\title{
Joint Scheduling and Resource Allocation in the OFDMA Downlink: Utility Maximization under Imperfect Channel-State Information
}

\author{
Rohit Aggarwal*, Mohamad Assaad ${ }^{\dagger}$, C. Emre Koksal ${ }^{*}$, and Philip Schniter* \\ *Dept. of ECE, The Ohio State University, Columbus, OH 43210. \\ Email: \{aggarwar,koksal,schniter\}@ece.osu.edu \\ †Dept. of Telecom., Supélec, France. \\ Email: mohamad.assaad@supelec.fr
}

\begin{abstract}
We consider the problem of simultaneous user-scheduling, power-allocation, and rate-selection in an OFDMA downlink, with the goal of maximizing expected sum-utility under a sum-power constraint. In doing so, we consider a family of generic goodput-based utilities that facilitate, e.g., throughput-based pricing, quality-of-service enforcement, and/or the treatment of practical modulation-and-coding schemes (MCS). Since perfect knowledge of channel state information (CSI) may be difficult to maintain at the base-station, especially when the number of users and/or subchannels is large, we consider scheduling and resource allocation under imperfect CSI, where the channel state is described by a generic probability distribution. First, we consider the "continuous" case where multiple users and/or code rates can timeshare a single OFDMA subchannel and time slot. This yields a non-convex optimization problem that we convert into a convex optimization problem and solve exactly using a dual optimization approach. Second, we consider the "discrete" case where only a single user and code rate is allowed per OFDMA subchannel per time slot. For the mixed-integer optimization problem that arises, we discuss the connections it has with the continuous case and show that it can solved exactly in some situations. For the other situations, we present a bound on the optimality gap. For both cases, we provide algorithmic implementations of the obtained solution. Finally, we study, numerically, the performance of the proposed algorithms under various degrees of CSI uncertainty, utilities, and OFDMA system configurations. In addition, we demonstrate advantages relative to existing state-of-the-art algorithms.
\end{abstract}




\section{INTRODUCTION}

In the downlink of a wireless orthogonal frequency division multiple access (OFDMA) system, the base station (BS) delivers data to a pool of users whose channels vary in both time and frequency. Since bandwidth and power resources are limited, the BS would like to allocate them most effectively, e.g., by pairing users with strong subchannels and distributing power in order to maximize some function of the delivered data rates [1]. Although, for resource allocation, one would ideally like to have access to instantaneous channel state information (CSI), such CSI is difficult to obtain in practice, and so resource allocation must be accomplished under imperfect CSI. Thus, in this paper, we consider simultaneous userscheduling, power-allocation, and rate-selection in an OFDMA downlink, given only a generic distribution for the subchannel signal-to-noise ratios (SNRs), with the goal of maximizing expected sum-utility under a sum-power constraint. In doing so, we consider relatively generic goodput-based utilities, facilitating, e.g., throughput-based pricing (e.g., [2]-[4]), quality-of-service enforcement, and/or the treatment of practical modulation-and-coding schemes (MCS).

In particular, we consider the above scheduling and resource allocation (SRA) problem under two scenarios. In the first scenario, we allow multiple users (and/or MCSs) to time-share any given subchannel and time-slot. In practice, this scenario occurs, e.g., in OFDMA systems where several users are multiplexed within a time-slot, such as IEEE 802.16/WiMAX [5] and 3GPP LTE [6]. Although the resulting optimization problem is non-convex, we show that it can be converted into a convex problem and solved exactly using a dual optimization approach. Based on a detailed analysis of the optimal solution, we propose a novel bisection-based algorithm that is faster than state-of-the-art golden-section based approaches (e.g., [7]) and that admits finite-iteration performance guarantees. In the second scenario, we allow at most one combination of user and MCS to be used on any given subchannel and time-slot. This scenario occurs widely in practice, such as in the Dedicated Traffic Channel (DTCH) mode of UMTS-LTE [8], and results in a mixed-integer optimization problem. Based on a detailed analysis of the optimal solution to this problem and its relationship to that in the first scenario, we propose a novel suboptimal algorithm that is faster than state-of-the-art golden-section and subgradient based approaches (e.g., [7], [9]), and we derive a novel tight bound on the optimality gap of our algorithm. Finally, we simulate our algorithms under various OFDMA system configurations, comparing against state-of-the-art approaches and genie-aided performance bounds.

We now discuss related work. The problem of OFDMA downlink SRA under perfect CSI has been studied in several papers, notably [10]-[15]. In [10], a utility maximization framework for discrete 
allocation was formulated to balance system efficiency and fairness, and efficient subgradient-based algorithms were proposed. In [11], a subchannel, rate, and power allocation algorithm was developed to minimize power consumption while maintaining a total rate-allocation requirement for every user. In [14], a weighted-sum capacity maximization problem with/without subchannel sharing was formulated to allocate subcarriers and powers. In [15], non-convex optimization problems regarding weighted sum-rate maximization and weighted sum-power minimization were solved using a Lagrange dual decomposition method. Compared to the above works, we extend the utility maximization framework to imperfect CSI and continuous allocations, and propose bisection-based algorithms that are faster for both the discrete and continuous allocation scenarios. Unlike [10]-[15], our utility framework can be applied to problems with/without fixed rate-power functions 1 . In additional, it can be applied to pricing-based utilities (e.g., responsive pricing and proportional fairness pricing) [2]. Furthermore, we study the relationship between the discrete and continuous allocation scenarios, and provide a tight bound on the duality gap of our proposed discrete-allocation scheme.

The problem of OFDMA downlink SRA under imperfect CSI was studied in several papers, notably [7], [9], [16], [17]. In [9], the authors considered the problem of discrete ergodic weighted sum-rate maximization for user scheduling and resource allocation, and studied the impact of channel estimation error due to pilot-aided MMSE channel estimation. In [7], a deterministic optimization problem was formulated using an upper bound on system capacity (via Jensen's inequality) as the objective. Both optimal and heuristic algorithms were then proposed to implement the obtained solution. Compared to these two works, we propose faster algorithms, applicable to a general utility maximization framework (of which the objectives in [7], [9] are special cases), under a more general class of channel estimators, and for both discrete and continuous subchannel allocations. Our algorithms are inspired by a rigorous analysis of the optimal solutions to the discrete and continuous problems. In [16], the problem of total transmit power minimization, subject to strict constraints on conditional expected user capacities, was investigated. In [17], the effect of heterogeneous delay requirements and outdated CSI on a particular discrete resource allocation problem was studied. In contrast, we consider a general utility maximization problem that allows us to attack problems that may or may not be based on fixed rate-power functions, as well as those based on pricing models. Relative to these works, we propose faster algorithms for both continuous and discrete allocation problems with provable bounds on their performances.

The remainder of this paper is organized as follows. In Section $\amalg$ we outline the system model and

\footnotetext{
${ }^{1}$ By a "fixed rate-power function" we mean that, for a given SNR, the achievable rate is a known function of the power.
} 
frame our optimization problems. In Section III we consider the "continuous" problem, where each subchannel can be shared by multiple users and rates, and find its exact solution. In Section IV] we consider the "discrete" problem, where each subchannel can support at most one combination of user and rate per time slot. In Section $\mathrm{V}$, we compare the performance of the proposed algorithms to reference algorithms under various settings. Finally, in Section VI] we conclude.

\section{System Model}

We consider a downlink OFDMA system with $N$ subchannels and $K$ active users $\left(N, K \in \mathbb{Z}^{+}\right)$as shown in Fig. 1, The scheduler-and-resource-allocator at the base-station uses the imperfect CSI to send data to the users, across OFDMA subchannels, in a way that maximizes utility. We assume that, for each user, there is an infinite backlog of data at the base-station, so that there is always data available to be transmitted. During every channel use and across every OFDMA subchannel, the base-station transmits codeword(s) from a generic signaling scheme, which propagate to the intended mobile recipient(s) through their respective fading channels. For a given user $k$, the OFDMA subchannels are assumed to be non-interfering, with gains that are time-invariant over each codeword duration and statistically independent of those for other users. Thus, the successful reception of a transmitted codeword depends on the corresponding subchannel's SNR $\gamma$, power $p$, and modulation and coding scheme (MCS), indexed by $m \in\{1, \ldots, M\}$. We assume that, for user $k$, MCS $m$ corresponds to a transmission rate of $r_{k, m}$ bits per codeword and a codeword error probability of $\epsilon_{k, m}(p \gamma)=a_{k, m} e^{-b_{k, m} p \gamma}$ for known constants $a_{k, m}$ and $b_{k, m}$ (see, e.g., [9]). Here, the subchannel SNR $\gamma$ is treated as an exogenous parameter, so that $p \gamma$ is the effective received SNR.

To precisely state our scheduling and resource allocation (SRA) problem, some additional notation is useful. To indicate how subchannels are partitioned among users and rates in each time-slot, we will use the proportionality indicator $I_{n, k, m}$, where $I_{n, k, m}=1$ means that subchannel $n$ is fully dedicated to user $k$ at MCS $m$, and $I_{n, k, m}=0$ means that subchannel $n$ is totally unavailable to user $k$ at MCS $m$. The subchannel resource constraint is then expressed as $\sum_{k, m} I_{n, k, m} \leq 1$ for all $n$. In the sequel, we consider two flavors of the SRA problem, a "continuous" one where each subchannel can be shared among multiple users and/or rates per time slot (i.e., $I_{n, k, m} \in[0,1]$ ), and a "discrete" one where each subchannel can be allocated to at most one user/rate combination per time slot (i.e., $I_{n, k, m} \in\{0,1\}$ ). We will use $p_{n, k, m} \geq 0$ as the power that would be expended on subchannel $n$ if it was fully allocated to the user/rate combination $(k, m)$. With this definition, the total expended power becomes $\sum_{n, k, m} I_{n, k, m} p_{n, k, m}$. Finally, we will use $\gamma_{n, k}$ to denote the $n^{\text {th }}$ subchannel's SNR for user $k$. Although we assume that the BS does not know the 
SNR realizations $\left\{\gamma_{n, k}\right\}$, we assume that it does know the (marginal) distribution of each $\gamma_{n, k}$.

When subchannel $n$ is fully dedicated to user $k$ with MCS $m$ and power $p_{n, k, m}$, the goodput $g_{n, k, m}=$ $\left(1-a_{k, m} e^{-b_{k, m} p_{n, k, m} \gamma_{n, k}}\right) r_{k, m}$ quantifies the expected number of bits, per codeword, transmitted without error. In the sequel, we focus on maximizing goodput-based utilities of the form $U_{n, k, m}\left(g_{n, k, m}\right)$, where $U_{n, k, m}(\cdot)$ is any generic real-valued function that is twice differentiable, strictly-increasing, and concave, with $U_{n, k, m}(0)<\infty$. (These conditions imply $U_{n, k, m}^{\prime}(\cdot)>0$ and $U_{n, k, m}^{\prime \prime}(\cdot) \leq 0$.) In particular, we aim to maximize the expected sum utility, $\mathrm{E}\left\{\sum_{n, k, m} I_{n, k, m} U_{n, k, m}\left(g_{n, k, m}\right)\right\}$, where the expectation is taken over the subchannel-SNRs $\left\{\gamma_{n, k}\right\}$ hidden within the goodputs. Incorporating a sum-power constraint of $P_{\text {con, }}$ our SRA problem becomes

$$
\begin{aligned}
& \mathrm{SRA} \triangleq \max _{\substack{\left\{p_{n, k, m} \geq 0\right\} \\
\left\{I_{n, k, m}\right\}}} \mathrm{E}\left\{\sum_{n=1}^{N} \sum_{k=1}^{K} \sum_{m=1}^{M} I_{n, k, m} U_{n, k, m}\left(\left(1-a_{k, m} e^{-b_{k, m} p_{n, k, m} \gamma_{n, k}}\right) r_{k, m}\right)\right\} \\
& \text { s.t. } \sum_{k, m} I_{n, k, m} \leq 1 \forall n \text { and } \sum_{n, k, m} I_{n, k, m} p_{n, k, m} \leq P_{\text {con. }} .
\end{aligned}
$$

The above formulation is sufficiently general to address a wide class of objectives. For example, to maximize sum-goodput, one would simply use $U_{n, k, m}(g)=g$. For weighted sum-goodput, one would instead choose $U_{n, k, m}(g)=w_{k} g$ with appropriately chosen weights $\left\{w_{k}\right\}$. To maximize weighted sum capacity $\sum_{n, k} w_{k} I_{n, k, 1} \log \left(1+p_{n, k, 1} \gamma_{n, k}\right)$, as in [9], one would choose $M=a_{k, 1}=b_{k, 1}=r_{k, 1}=1$, and set $U_{n, k, 1}(g)=w_{k} \log (1-\log (1-g))$ for $g \in[0,1)$. Commonly used utilities constructed from concave functions of capacity $\log \left(1+p_{n, k, 1} \gamma_{n, k}\right)$, such as max-min fairness and the utilities in [10] and [7], can also be handled by our formulation. For example, the utility $U_{n, k, m}(g)=1-e^{-w_{k} g}$ (for some positive $\left.\left\{w_{k}\right\}\right)$ is appropriate for "elastic" applications such as file transfer [3], [4]. Our formulation also supports various pricing models [2], such as flat-pricing, responsive pricing, proportional fairness pricing, and effective-bandwidth pricing.

Next, in Section III, we study the SRA problem for the continuous case $I_{n, k, m} \in[0,1]$, and in Section IV we study it for the discrete case $I_{n, k, m} \in\{0,1\}$.

\section{Optimal Scheduling AND Resource Allocation with SUbChannel SHARing}

In this section, we address the SRA problem in the case where $I_{n, k, m} \in[0,1] \forall(n, k, m)$. Recall that this problem arises when sharing of any subchannel by multiple users and/or multiple MCS combinations is allowed. We refer to this problem as the "continuous scheduling and resource allocation" (CSRA) problem. Defining $\boldsymbol{I}$ as the $N \times K \times M$ matrix with $(n, k, m)^{\text {th }}$ element as $I_{n, k, m}$ and the domain of $\boldsymbol{I}$ 
as

$$
\mathcal{I}_{\text {CSRA }}:=\left\{\boldsymbol{I}: \boldsymbol{I} \in[0,1]^{N \times K \times M}, \quad \sum_{k, m} I_{n, k, m} \leq 1 \forall n\right\},
$$

the CSRA problem can be stated as

CSRA $:=\min _{\substack{\left\{p_{n, k, m} \geq 0\right\} \\ \boldsymbol{I} \in \mathcal{I}_{\text {CSRA }}}}-\sum_{n, k, m} I_{n, k, m} \mathrm{E}\left\{U_{n, k, m}\left(\left(1-a_{k, m} e^{-b_{k, m} p_{n, k, m} \gamma_{n, k}}\right) r_{k, m}\right)\right\}$ s.t. $\sum_{n, k, m} I_{n, k, m} p_{n, k, m} \leq P_{\text {con }}$ (2)

This problem has a non-convex constraint set, making it a non-convex optimization problem. In order to convert it into a convex optimization problem, we write the "actual" power allocated to user $k$ at MCS $m$ on subchannel $n$ as $x_{n, k, m}=I_{n, k, m} p_{n, k, m}$. Then, the problem becomes

$$
\mathrm{CSRA}=\min _{\substack{\left\{x_{n, k, m} \geq 0\right\} \\ \boldsymbol{I} \in \mathcal{I}_{\mathrm{CSRA}}}} \sum_{n, k, m} I_{n, k, m} F_{n, k, m}\left(I_{n, k, m}, x_{n, k, m}\right) \quad \text { s.t. } \sum_{n, k, m} x_{n, k, m} \leq P_{\text {con }},
$$

where $F_{n, k, m}(\cdot, \cdot)$ is given by

$$
F_{n, k, m}\left(I_{n, k, m}, x_{n, k, m}\right)= \begin{cases}-\mathrm{E}\left\{U_{n, k, m}\left(\left(1-a_{k, m} e^{-b_{k, m} x_{n, k, m} \gamma_{n, k} / I_{n, k, m}}\right) r_{k, m}\right)\right\} & \text { if } I_{n, k, m} \neq 0 \\ 0 & \text { otherwise }\end{cases}
$$

The modified problem in (3) is a convex optimization problem with a convex objective function and linear inequality constraint. Moreover, Slater's condition is satisfied at $I_{n, k, m}=\frac{1}{2 K M}$ and $x_{n, k, m}=$ $\frac{P_{\mathrm{con}}}{N} I_{n, k, m}, \forall n, k, m$. Hence, the solution of (3) is the same as that of its dual problem (i.e., zero duality gap) [18]. Let us denote the optimal $\boldsymbol{I}$ and $\boldsymbol{x}$ for (3) by $\boldsymbol{I}_{\mathrm{CSRA}}^{*}$ and $\boldsymbol{x}_{\mathrm{CSRA}}^{*}$, respectively, and let $\boldsymbol{p}_{\mathrm{CSRA}}^{*}$ be the corresponding $\boldsymbol{p}$.

Writing the dual formulation, using $\mu$ as the dual variable, the Lagrangian of (3) is

$$
L(\mu, \boldsymbol{I}, \boldsymbol{x})=\sum_{n, k, m} I_{n, k, m} F_{n, k, m}\left(I_{n, k, m}, x_{n, k, m}\right)+\left(\sum_{n, k, m} x_{n, k, m}-P_{\text {con }}\right) \mu,
$$

where we use $\boldsymbol{x}$ to denote the $N \times K \times M$ matrix $\left[x_{n, k, m}\right]$. The corresponding unconstrained dual problem, then, becomes

$$
\begin{aligned}
& \max _{\mu \geq 0} \min _{\substack{\boldsymbol{x} \succeq 0 \\
\boldsymbol{I} \in \mathcal{I}_{\text {CSRA }}}} L(\mu, \boldsymbol{I}, \boldsymbol{x}) \\
& =\max _{\mu \geq 0} \min _{\boldsymbol{I} \in \mathcal{I}_{\text {CSRA }}} L\left(\mu, \boldsymbol{I}, \boldsymbol{x}^{*}(\mu, \boldsymbol{I})\right)=\max _{\mu \geq 0} L\left(\mu, \boldsymbol{I}^{*}(\mu), \boldsymbol{x}^{*}\left(\mu, \boldsymbol{I}^{*}(\mu)\right)\right)=L\left(\mu^{*}, \boldsymbol{I}^{*}\left(\mu^{*}\right), \boldsymbol{x}^{*}\left(\mu^{*}, \boldsymbol{I}^{*}\left(\mu^{*}\right)\right)\right),
\end{aligned}
$$

where $\boldsymbol{x} \succeq 0$ means that $x_{n, k, m} \geq 0 \forall n, k, m, \boldsymbol{x}^{*}(\mu, \boldsymbol{I})$ denotes the optimal $\boldsymbol{x}$ for a given $\mu$ and $\boldsymbol{I}$, $\boldsymbol{I}^{*}(\mu) \in \mathcal{I}_{\text {CSRA }}$ denotes the optimal $\boldsymbol{I}$ for a given $\mu$, and $\mu^{*}$ denotes the optimal $\mu$.

In the next few subsections, we will optimize the Lagrangian according to (6) w.r.t. $\boldsymbol{x}, \boldsymbol{I}$, and $\mu$

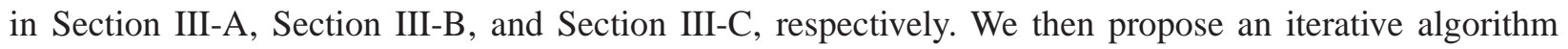
to solve CSRA problem in Section [II-D Finally, we discuss some important properties of the CSRA solution in Section 


\section{A. Optimizing over total powers, $\boldsymbol{x}$, for a given $\mu$ and user-MCS allocation matrix $\boldsymbol{I}$}

The Lagrangian in (5) is a convex function of $\boldsymbol{x}$. Therefore, any local minimum of the function is a global minimum. Calculating the derivative of $L(\mu, \boldsymbol{I}, \boldsymbol{x})$ w.r.t. $x_{n, k, m}$, we get

$$
\frac{\partial L(\mu, \boldsymbol{I}, \boldsymbol{x})}{\partial x_{n, k, m}}= \begin{cases}\mu & \text { if } I_{n, k, m}=0 \\ \mu & -a_{k, m} b_{k, m} r_{k, m} \mathrm{E}\left\{U _ { n , k , m } ^ { \prime } \left(\left(1-a_{k, m} e^{-b_{k, m} x_{n, k, m} \gamma_{n, k} / I_{n, k, m}}\right)\right.\right. \\ \left.\left.\quad \times r_{k, m}\right) \gamma_{n, k} e^{-b_{k, m} x_{n, k, m} \gamma_{n, k} / I_{n, k, m}}\right\} & \text { otherwise. }\end{cases}
$$

Clearly, if $I_{n, k, m}=0$, then $L(\cdot, \cdot, \cdot)$ is an increasing 2 function of $x_{n, k, m}$ since $\mu \geq 0$. Therefore, $x_{n, k, m}^{*}(\mu, \boldsymbol{I})=0$. But if $I_{n, k, m} \neq 0$, then $\frac{\partial L(\mu, \boldsymbol{I}, \boldsymbol{x})}{\partial x_{n, k, m}}$ is an increasing function of $x_{n, k, m}$ since $U_{n, k, m}^{\prime}(\cdot)$ is a decreasing function of $x_{n, k, m}$. Thus, we have

$$
\mu-a_{k, m} b_{k, m} r_{k, m} \mathrm{E}\left\{U_{n, k, m}^{\prime}\left(\left(1-a_{k, m} e^{-b_{k, m} x_{n, k, m} \gamma_{n, k} / I_{n, k, m}}\right) r_{k, m}\right) \gamma_{n, k} e^{-b_{k, m} x_{n, k, m} \gamma_{n, k} / I_{n, k, m}}\right\}=0
$$

for some positive $x_{n, k, m}$ if and only if $0 \leq \mu \leq a_{k, m} b_{k, m} r_{k, m} U_{n, k, m}^{\prime}\left(\left(1-a_{k, m}\right) r_{k, m}\right) \mathrm{E}\left\{\gamma_{n, k}\right\}$. Therefore,

$$
x_{n, k, m}^{*}(\mu, \boldsymbol{I})= \begin{cases}\tilde{x}_{n, k, m}(\mu, \boldsymbol{I}) & \text { if } 0 \leq \mu \leq a_{k, m} b_{k, m} r_{k, m} U_{n, k, m}^{\prime}\left(\left(1-a_{k, m}\right) r_{k, m}\right) \mathrm{E}\left\{\gamma_{n, k}\right\} \\ 0 & \text { otherwise, }\end{cases}
$$

where $\tilde{x}_{n, k, m}(\mu, \boldsymbol{I})$ satisfies

$\mu=a_{k, m} b_{k, m} r_{k, m} \mathrm{E}\left\{U_{n, k, m}^{\prime}\left(\left(1-a_{k, m} e^{-b_{k, m} \tilde{x}_{n, k, m}(\mu, \boldsymbol{I}) \gamma_{n, k} / I_{n, k, m}}\right) r_{k, m}\right) \gamma_{n, k} e^{-b_{k, m} \tilde{x}_{n, k, m}(\mu, \boldsymbol{I}) \gamma_{n, k} / I_{n, k, m}}\right\}$.

From (10), we observe that $\tilde{x}_{n, k, m}(\mu, \boldsymbol{I})=\tilde{p}_{n, k, m}(\mu) I_{n, k, m}$, where $\tilde{p}_{n, k, m}(\mu)$ satisfies

$$
\mu=a_{k, m} b_{k, m} r_{k, m} \mathrm{E}\left\{U_{n, k, m}^{\prime}\left(\left(1-a_{k, m} e^{-b_{k, m} \tilde{p}_{n, k, m}(\mu) \gamma_{n, k}}\right) r_{k, m}\right) \gamma_{n, k} e^{-b_{k, m} \tilde{p}_{n, k, m}(\mu) \gamma_{n, k}}\right\} .
$$

Combining the above observations, we can write for any $\boldsymbol{I} \in \mathcal{I}_{\mathrm{CSRA}}$ and $(n, k, m)$ that

where

$$
x_{n, k, m}^{*}(\mu, \boldsymbol{I})=I_{n, k, m} p_{n, k, m}^{*}(\mu),
$$

$$
p_{n, k, m}^{*}(\mu)= \begin{cases}\tilde{p}_{n, k, m}(\mu) & \text { if } 0 \leq \mu \leq a_{k, m} b_{k, m} r_{k, m} U_{n, k, m}^{\prime}\left(\left(1-a_{k, m}\right) r_{k, m}\right) \mathrm{E}\left\{\gamma_{n, k}\right\} \\ 0 & \text { otherwise, }\end{cases}
$$

and $\tilde{p}_{n, k, m}(\mu)$ satisfies (11). Note that if such a $\tilde{p}_{n, k, m}(\mu)$ exists that satisfies (11), then it is unique. This is because, in (11),$U_{n, k, m}^{\prime}(\cdot)$ is a continuous decreasing positive function and $e^{-b_{k, m} \tilde{p}_{n, k, m}(\mu) \gamma_{n, k}}$

${ }^{2}$ We use the terms "increasing" and "decreasing" interchangeably with "non-decreasing" and "non-increasing", respectively. The terms "strictly-increasing" and "strictly-decreasing" are used when appropriate. 


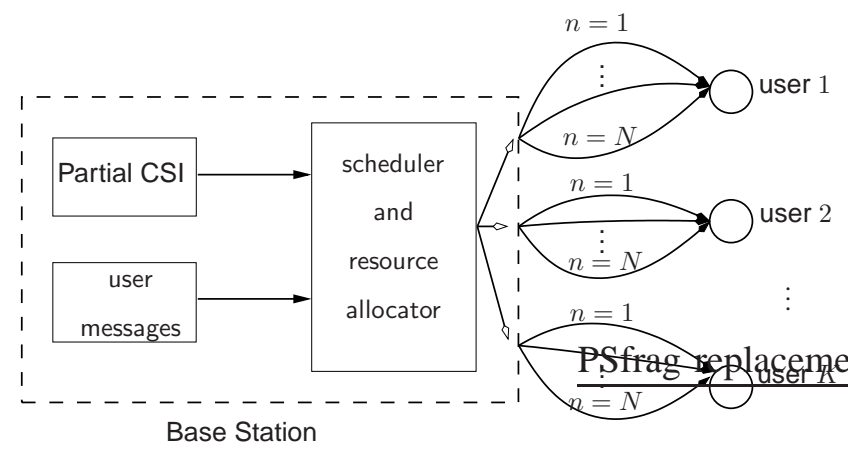

Fig. 1: System model of a downlink OFDMA system with $N$ subchannels and $K$ users. Here, $n$ is the subchannel index.

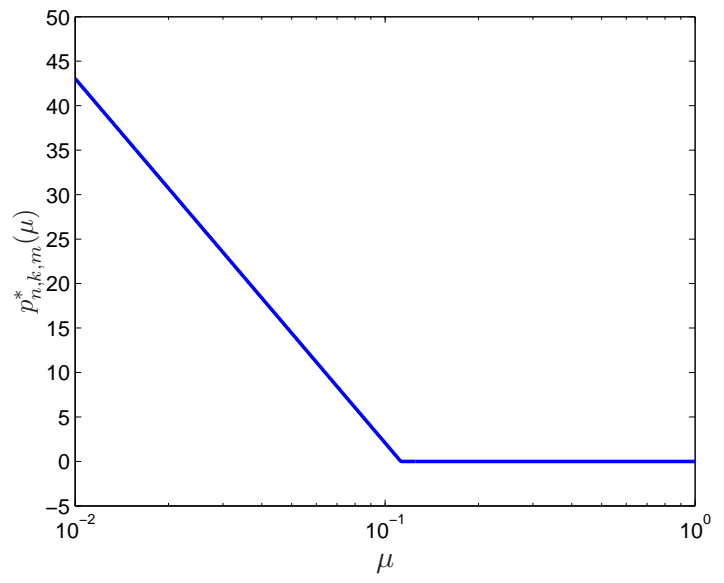

Fig. 2: Prototypical plot of $p_{n, k, m}^{*}(\mu)$ as a function of $\mu$. The choice of system parameters are the same as those used in Section $\mathrm{V}$

is a strictly-decreasing continuous function of $\tilde{p}_{n, k, m}(\mu)$, which makes the right side of (11) a strictlydecreasing continuous function of $\tilde{p}_{n, k, m}(\mu)$. Therefore, in the domain of its existence, $\tilde{p}_{n, k, m}(\mu)$ is unique and decreases continuously with increase in $\mu$. Consequently, $x_{n, k, m}^{*}(\mu, \boldsymbol{I})$ is a decreasing continuous function of $\mu$. Figure 2 shows an example of the variation of $p_{n, k, m}^{*}(\mu)$ w.r.t. $\mu$.

\section{B. Optimizing over user-MCS allocation matrix I for a given $\mu$}

Substituting $\boldsymbol{x}^{*}(\mu, \boldsymbol{I})$ from (12) into (5), we get the Lagrangian

$$
\begin{aligned}
& L\left(\mu, \boldsymbol{I}, \boldsymbol{x}^{*}(\mu, \boldsymbol{I})\right) \\
& =-\mu P_{\mathrm{con}}+\sum_{n} \underbrace{\sum_{k, m} I_{n, k, m}[\overbrace{-\mathrm{E}\left\{U _ { n , k , m } \left(\left(1-a_{k, m} e^{\left.\left.\left.-b_{k, m} p_{n, k, m}^{*}(\mu) \gamma_{n, k}\right) r_{k, m}\right)\right\}+\mu p_{n, k, m}^{*}(\mu)}\right]\right.\right.}^{V_{n, k, m}\left(\mu, p_{n, k, m}^{*}(\mu)\right)}]}_{\sum_{n}\left(\mu, \boldsymbol{I}_{n}\right)},(14)
\end{aligned}
$$

where $\boldsymbol{I}_{n}=\left\{I_{n, k, m} \forall(k, m)\right\}$. Since the above Lagrangian contains the sum of $L_{n}\left(\mu, \boldsymbol{I}_{n}\right)$ over $n$, minimizing $L_{n}\left(\mu, \boldsymbol{I}_{n}\right)$ for every $n$ (over all possible $\boldsymbol{I}_{n}$ ) minimizes the Lagrangian. Recall that $L_{n}\left(\mu, \boldsymbol{I}_{n}\right)$ is a linear function of $\left\{I_{n, k, m} \forall(k, m)\right\}$ that satisfies $\sum_{k, m} I_{n, k, m} \leq 1$. Therefore, $L_{n}\left(\mu, \boldsymbol{I}_{n}\right)$ is minimized by the $\boldsymbol{I}_{n}$ that gives maximum possible weight to the $(k, m)$ combination with the most negative value of $V_{n, k, m}\left(\mu, p_{n, k, m}^{*}(\mu)\right)$. To write this mathematically, let us define, for each $\mu$ and subchannel $n$, a set of participating user-MCS combinations that yield the same most-negative value of $V_{n, k, m}\left(\mu, p_{n, k, m}^{*}(\mu)\right)$ over all $(k, m)$ as follows:

$$
S_{n}(\mu) \triangleq\left\{(k, m):(k, m)=\underset{\left(k^{\prime}, m^{\prime}\right)}{\operatorname{argmin}} V_{n, k^{\prime}, m^{\prime}}\left(\mu, p_{n, k^{\prime}, m^{\prime}}^{*}(\mu)\right), \text { and } V_{n, k, m}\left(\mu, p_{n, k, m}^{*}(\mu)\right) \leq 0\right\} .
$$


If $S_{n}(\mu)$ is a null or a singleton set, then the optimal allocation on subchannel $n$ is given by

$$
I_{n, k, m}^{*}(\mu)= \begin{cases}1 & \text { if }(k, m) \in S_{n}(\mu) \\ 0 & \text { otherwise. }\end{cases}
$$

However, if $\left|S_{n}(\mu)\right|>1$ (where $\left|S_{n}(\mu)\right|$ denotes the cardinality of $S_{n}(\mu)$ ), then multiple $(k, m)$ combinations contribute equally towards the minimum value of $L_{n}(\mu, \boldsymbol{I})$, and thus the optimum can be reached by sharing subchannel $n$. In particular, let us suppose that $S_{n}(\mu)=\left\{\left(k_{1}(n), m_{1}(n)\right), \ldots,\left(k_{\left|S_{n}(\mu)\right|}(n), m_{\left|S_{n}(\mu)\right|}(n)\right)\right\}$. Then, the optimal allocation of subchannel $n$ is given by

$$
I_{n, k, m}^{*}(\mu)= \begin{cases}I_{n, k_{i}(n), m_{i}(n)} & \text { if }(k, m)=\left(k_{i}(n), m_{i}(n)\right) \text { for some } i \in\left\{1, \ldots,\left|S_{n}(\mu)\right|\right\} \\ 0 & \text { otherwise, }\end{cases}
$$

where the vector $\left(I_{n, k_{1}(n), m_{1}(n)}, \ldots, I_{n, k_{\left|S_{n}(\mu)\right|}(n), m_{\left|S_{n}(\mu)\right|}(n)}\right)$ is any point in the unit- $\left(\left|S_{n}(\mu)\right|-1\right)$ simplex, i.e., it belongs to the space $[0,1]^{\left|S_{n}(\mu)\right|}$ and satisfies

$$
\sum_{i=1}^{\left|S_{n}(\mu)\right|} I_{n, k_{i}(n), m_{i}(n)}=1 \text {. }
$$

\section{Optimizing over $\mu$}

In order to optimize over $\mu$, we can calculate the Lagrangian optimized for a given value of $\mu$ as

$$
\begin{aligned}
& L\left(\mu, \boldsymbol{I}^{*}(\mu), \boldsymbol{x}^{*}\left(\mu, \boldsymbol{I}^{*}(\mu)\right)\right) \\
& =-\mu P_{\mathrm{con}}+\sum_{n, k, m} I_{n, k, m}^{*}(\mu)\left[-\mathrm{E}\left\{U_{n, k, m}\left(\left(1-a_{k, m} e^{-b_{k, m} p_{n, k, m}^{*}(\mu) \gamma_{n, k}}\right) r_{k, m}\right)\right\}+\mu p_{n, k, m}^{*}(\mu)\right],(19)
\end{aligned}
$$

and then maximize it over all possible values of $\mu \geq 0$ to find $\mu^{*}$. Notice from (16) (18) that we have $\sum_{k, m} I_{n, k, m}^{*}\left(\mu^{*}\right)=1$ for at least one $n$. Otherwise, $\boldsymbol{I}^{*}\left(\mu^{*}\right)=\mathbf{0}$ which, clearly, is not the optimal solution. Therefore, $\mu^{*} \geq \mu_{\min }>0$, where

$$
\mu_{\min }=\min _{n, k, m} a_{k, m} b_{k, m} r_{k, m} \mathrm{E}\left\{U_{n, k, m}^{\prime}\left(\left(1-a_{k, m} e^{-b_{k, m} P_{\mathrm{con}} \gamma_{n, k}}\right) r_{k, m}\right) \gamma_{n, k} e^{-b_{k, m} P_{\mathrm{con}} \gamma_{n, k}}\right\}
$$

is obtained by taking $\tilde{p}_{n, k, m}(\mu) \rightarrow P_{\text {con }}$ for all $(n, k, m)$ in the right side of (11). Since $p_{n, k, m}^{*}(\mu)$ is a decreasing continuous function of $\mu$ (seen in Section $\amalg$ II-A), we have $\sum_{n, k, m} x_{n, k, m}^{*}(\mu, \boldsymbol{I})>P_{\text {con }}$ for all $\boldsymbol{I} \neq \mathbf{0}$ and $\mu<\mu_{\min }$. We can also obtain an upper bound $\mu^{*} \leq \mu_{\max }$, where

$$
\mu_{\max }=\max _{n, k, m} a_{k, m} b_{k, m} r_{k, m} U_{n, k, m}^{\prime}\left(\left(1-a_{k, m}\right) r_{k, m}\right) \mathrm{E}\left\{\gamma_{n, k}\right\}
$$

is obtained by taking $\tilde{p}_{n, k, m}(\mu) \rightarrow 0$ in the right side of (11). Thus, for any $\mu>\mu_{\max }$, we have that $x_{n, k, m}^{*}(\mu, \boldsymbol{I})=0 \forall n, k, m, \boldsymbol{I}$. Since the primal objective in (3) is certainly not maximized when zero power is allocated on all subchannels, we have $\mu^{*} \in\left[\mu_{\min }, \mu_{\max }\right] \subset(0, \infty)$. 
At the optimal $\mu$, i.e., $\mu^{*}$, if we have $\left|S_{n}\left(\mu^{*}\right)\right| \leq 1 \forall n$, then the optimal CSRA allocation, $\boldsymbol{I}_{\text {CSRA }}^{*}$, equals $\boldsymbol{I}^{*}\left(\mu^{*}\right)$ and can be calculated using (16). Moreover, the optimal power allocation $\boldsymbol{p}_{\mathrm{CSRA}}^{*}$ allocates

$$
p_{n, k, m, \mathrm{CSRA}}^{*}= \begin{cases}p_{n, k, m}^{*}\left(\mu^{*}\right) & \text { if } I_{n, k, m}^{*}\left(\mu^{*}\right) \neq 0 \\ 0 & \text { otherwise }\end{cases}
$$

to every possible $(n, k, m)$ combination. However, if for some $n$, we have $\left|S_{n}\left(\mu^{*}\right)\right|>1$, then ambiguity arises due to multiple possibilities of $\boldsymbol{I}^{*}\left(\mu^{*}\right)$ obtained via (17). In order to find the optimal user-MCS allocation in such cases, we use the fact that the CSRA problem in (3) is a convex optimization problem whose exact solution satisfies the sum-power constraint with equality, i.e.,

$$
\sum_{n, k, m} x_{n, k, m}^{*}\left(\mu^{*}, \boldsymbol{I}^{*}\left(\mu^{*}\right)\right)=\sum_{n, k, m} I_{n, k, m}^{*}\left(\mu^{*}\right) p_{n, k, m}^{*}\left(\mu^{*}\right)=P_{\text {con }}
$$

This is because $\mu^{*} \geq \mu_{\min }>0$ (shown earlier) and the complementary slackness condition gives that $\mu^{*}\left(\sum_{n, k, m} x_{n, k, m}^{*}\left(\mu^{*}, \boldsymbol{I}^{*}\left(\mu^{*}\right)\right)-P_{\mathrm{con}}\right)=0$. Now, recall that the total power allocated to any subchannel $n$ at $\mu^{*}$ is $\sum_{i=1}^{\left|S_{n}\left(\mu^{*}\right)\right|} I_{n, k_{i}(n), m_{i}(n)} p_{n, k_{i}(n), m_{i}(n)}^{*}\left(\mu^{*}\right)$ where $\left\{I_{n, k_{i}(n), m_{i}(n)}\right\}_{i=1}^{\left|S_{n}\left(\mu^{*}\right)\right|}$ satisfies (18). This quantity is dependent on the choice of values for $\left\{I_{n, k_{i}(n), m_{i}(n)}\right\}_{i=1}^{\left|S_{n}\left(\mu^{*}\right)\right|}$ and takes on any value between an upper and lower bound given by the following equation:

$$
\min _{i} p_{n, k_{i}(n), m_{i}(n)}^{*}\left(\mu^{*}\right) \leq \sum_{i=1}^{\left|S_{n}\left(\mu^{*}\right)\right|} I_{n, k_{i}(n), m_{i}(n)} p_{n, k_{i}(n), m_{i}(n)}^{*}\left(\mu^{*}\right) \leq \max _{i} p_{n, k_{i}(n), m_{i}(n)}^{*}\left(\mu^{*}\right) .
$$

Note that the existence of at least one $\boldsymbol{I}=\boldsymbol{I}^{*}\left(\mu^{*}\right)$ satisfying

$$
\sum_{n} \sum_{i} I_{n, k_{i}(n), m_{i}(n)} p_{n, k_{i}(n), m_{i}(n)}^{*}\left(\mu^{*}\right)=P_{\text {con }}
$$

is guaranteed by the optimality of the dual solution (of our convex CSRA problem over a closed constraint set). Therefore, we necessarily have $\sum_{n} \min _{i} p_{n, k_{i}(n), m_{i}(n)}^{*}\left(\mu^{*}\right) \leq P_{\text {con }}$, and $\sum_{n} \max _{i} p_{n, k_{i}(n), m_{i}(n)}^{*}\left(\mu^{*}\right) \geq$ $P_{\text {con }}$. In addition, all choices of user-MCS allocations, $I^{*}\left(\mu^{*}\right)$, given by (17) that satisfy the equality

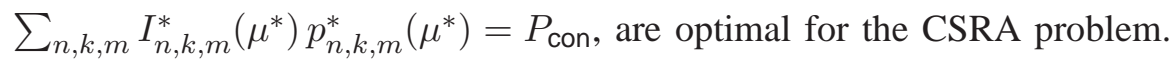

In the case that the optimal solution $\boldsymbol{I}^{*}\left(\mu^{*}\right)$ is non-unique, i.e., $\left|S_{n}\left(\mu^{*}\right)\right|>1$ for some $n$, then one instance of $\boldsymbol{I}^{*}\left(\mu^{*}\right)$ can be found as follows. For each subchannel $n$, define

$$
\begin{aligned}
\left(k_{\max }\left(n, \mu^{*}\right), m_{\max }\left(n, \mu^{*}\right)\right) & :=\underset{i}{\operatorname{argmax}} p_{n, k_{i}(n), m_{i}(n)}^{*}\left(\mu^{*}\right), \\
\left(k_{\min }\left(n, \mu^{*}\right), m_{\min }\left(n, \mu^{*}\right)\right) & :=\underset{i}{\operatorname{argmin}} p_{n, k_{i}(n), m_{i}(n)}^{*}\left(\mu^{*}\right),
\end{aligned}
$$

and find the value of $\lambda \in[0,1]$ for which

$$
\lambda\left(\sum_{n} p_{n, k_{\min }\left(n, \mu^{*}\right), m_{\min }\left(n, \mu^{*}\right)}\left(\mu^{*}\right)\right)+(1-\lambda)\left(\sum_{n} p_{n, k_{\max }\left(n, \mu^{*}\right), m_{\max }\left(n, \mu^{*}\right)}\left(\mu^{*}\right)\right)=P_{\text {con }},
$$


i.e.,

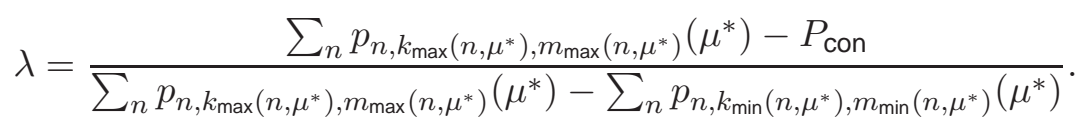

Now, defining two specific allocations, $\boldsymbol{I}^{\min }\left(\mu^{*}\right)$ and $\boldsymbol{I}^{\max }\left(\mu^{*}\right)$, as

$I_{n, k, m}^{\min }\left(\mu^{*}\right)=\left\{\begin{array}{ll}1 & (k, m)=\left(k_{\min }\left(n, \mu^{*}\right), m_{\min }\left(n, \mu^{*}\right)\right) \\ 0 & \text { otherwise }\end{array}, I_{n, k, m}^{\max }\left(\mu^{*}\right)= \begin{cases}1 & (k, m)=\left(k_{\max }\left(n, \mu^{*}\right), m_{\max }\left(n, \mu^{*}\right)\right) \\ 0 & \text { otherwise }\end{cases}\right.$

respectively, the optimal user-MCS allocation is given by $\boldsymbol{I}_{\text {CSRA }}^{*}=\lambda \boldsymbol{I}^{\min }\left(\mu^{*}\right)+(1-\lambda) \boldsymbol{I}^{\max }\left(\mu^{*}\right)$. The corresponding optimal power allocation is then given by (22). It can be seen that this solution satisfies the subchannel constraint as well as the sum power constraint with equality, i.e.,

$$
\sum_{n, k, m} I_{n, k, m}^{*}\left(\mu^{*}\right) p_{n, k, m}^{*}\left(\mu^{*}\right)=\sum_{n, k, m} x_{n, k, m}^{*}\left(\mu^{*}, \boldsymbol{I}^{*}\left(\mu^{*}\right)\right)=P_{\text {con } .}
$$

Two interesting observations can be made from the above discussion. Firstly, for any choice of concave utility functions $U_{n, k, m}(\cdot)$, there exists an optimal scheduling and resource allocation strategy that allocates each subchannel to at most 2 user-MCS combinations. Therefore, when allocating $N$ subchannels, even if more than $2 N$ user-MCS options are available, at most $2 N$ such options will be used. Secondly, if $\boldsymbol{I}^{\min }\left(\mu^{*}\right)=\boldsymbol{I}^{\max }\left(\mu^{*}\right)$, then the exact CSRA solution allocates power to at most one $(k, m)$ combination for every subchannel, i.e., no subchannel is shared among any two or more user-MCS combinations. This observation will motivate the SRA problem's solution without subchannel sharing in Section IV

\section{Algorithmic implementation}

In practice, it is not possible to search exhaustively over $\mu \in\left[\mu_{\min }, \mu_{\max }\right]$. Thus, we propose an algorithm to reach solutions in close (and adjustable) proximity to the optimal. The algorithm first narrows down the location of $\mu^{*}$ using a bisection-search over $\left[\mu_{\min }, \mu_{\max }\right]$ for the optimum total power allocation, and then finds a set of resource allocations $(\boldsymbol{I}, \boldsymbol{x})$ that achieve a total utility close to the optimal.

To proceed in this direction, with the aim of developing a framework to do bisection-search over $\mu$, let us define the total optimal allocated power for a given value of $\mu$ as follows:

$$
X_{\text {tot }}^{*}(\mu) \triangleq \sum_{n, k, m} x_{n, k, m}^{*}\left(\mu, \boldsymbol{I}^{*}(\mu)\right),
$$

where $\boldsymbol{I}^{*}(\mu)$ and $\boldsymbol{x}^{*}\left(\mu, \boldsymbol{I}^{*}(\mu)\right)$ (defined in (6) ) minimize the Lagrangian (defined in (5)) for a given $\mu$. The following lemma relates the variation of $X_{\text {tot }}^{*}(\mu)$ with respect to $\mu$.

Lemma 1. The total optimal power allocation, $X_{\text {tot }}^{*}(\mu)$, is a monotonically decreasing function of $\mu$. 
Proof: A proof sketch is given in Appendix B For the full proof, see [19].

A sample plot of $X_{\text {tot }}^{*}(\mu)$ and $L\left(\mu, \boldsymbol{I}^{*}(\mu), \boldsymbol{x}^{*}\left(\mu, \boldsymbol{I}^{*}(\mu)\right)\right)$ as a function of $\mu$ is shown in Figure 3 From the figure, three observations can be made. First, as $\mu$ increases, the optimal total allocated power decreases, as expected from Lemma 1. Second, as expected, the Lagrangian is maximized for that value

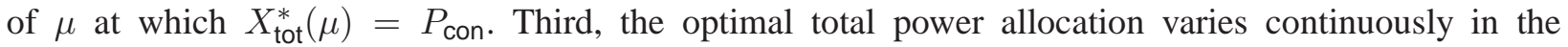
region of $\mu$ where the optimal allocation, $\boldsymbol{I}^{*}(\mu)$, remains constant and takes a jump (negative) when $\boldsymbol{I}^{*}(\mu)$ changes. This happens for the following reason. We know, for any $(n, k, m)$, that $p_{n, k, m}^{*}(\mu)$ is a continuous function of $\mu$. Thus, when the optimal allocation remains constant over a range of $\mu$, the total power allocated, $\sum_{n, k, m} I_{n, k, m}^{*}(\mu) p_{n, k, m}^{*}(\mu)$ also varies continuously with $\mu$. However, at the point of discontinuity ( say $\tilde{\mu}$ ), multiple optimal allocations achieve the same optimal value of Lagrangian. In other words, $\left|S_{n}(\tilde{\mu})\right|>1$ for some $n$. In that case, $X_{\text {tot }}^{*}(\tilde{\mu})$ can take any value in the interval

$$
\left[\sum_{n} p_{n, k_{\min }(n), m_{\min }(n)}^{*}(\tilde{\mu}), \quad \sum_{n} p_{n, k_{\max }(n), m_{\max }(n)}^{*}(\tilde{\mu})\right]
$$

while achieving the same minimum value of the Lagrangian at $\tilde{\mu}$. Applying Lemma 1 , we have

$$
X_{\mathrm{tot}}^{*}\left(\tilde{\mu}-\Delta_{1}\right) \geq \sum_{n} p_{n, k_{\max }(n), m_{\max }(n)}^{*}(\tilde{\mu}) \geq X_{\mathrm{tot}}^{*}(\tilde{\mu}) \geq \sum_{n} p_{n, k_{\min }(n), m_{\min }(n)}^{*}(\tilde{\mu}) \geq X_{\mathrm{tot}}^{*}\left(\tilde{\mu}+\Delta_{2}\right)
$$

for any $\Delta_{1}, \Delta_{2}>0$, causing a jump of $\left(\sum_{n} p_{n, k_{\min }(n), m_{\min }(n)}^{*}(\tilde{\mu})-\sum_{n} p_{n, k_{\max }(n), m_{\max }(n)}^{*}(\tilde{\mu})\right)$ in the total optimal power allocation at $\tilde{\mu}$.

Lemma 1 allows us to do a bisection-search over $\mu$ since $X_{\text {tot }}^{*}(\mu)$ is a decreasing function of $\mu$ and

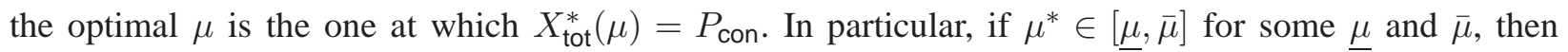
$\mu^{*} \in\left[\frac{\underline{\underline{\mu}}+\bar{\mu}}{2}, \bar{\mu}\right]$ if $X_{\text {tot }}^{*}\left(\frac{\underline{\mu}+\bar{\mu}}{2}\right)>P_{\text {con }}$, otherwise $\mu^{*} \in\left[\underline{\mu}, \frac{\underline{\mu}+\bar{\mu}}{2}\right]$. Using this concept, we propose an algorithm in Table \ that finds an interval $[\underline{\mu}, \bar{\mu}]$, such that $\mu^{*} \in[\underline{\mu}, \bar{\mu}]$ and $\bar{\mu}-\underline{\mu} \leq \kappa$, where $\kappa(>0)$ is a tuning-parameter, and allocates resources based on optimal resource allocations at $\underline{\mu}$ and $\bar{\mu}$.

The following lemma characterizes the relationship between the tuning parameter $\kappa$ and the accuracy of the obtained solution.

Lemma 2. Let $\mu^{*} \in[\underline{\mu}, \bar{\mu}]$ be the point where the proposed CSRA algorithm stops, and the total utility obtained by the proposed algorithm and the exact CSRA solution be $\hat{U}_{\mathrm{CSRA}}(\underline{\mu}, \bar{\mu})$ and $U_{\mathrm{CSRA}}^{*}$, respectively. Then, $0 \leq U_{\text {CSRA }}^{*}-\hat{U}_{\text {CSRA }}(\underline{\mu}, \bar{\mu}) \leq(\bar{\mu}-\underline{\mu}) P_{\text {con }}$.

Proof: For proof, see Appendix [C]

Since our algorithm stops when $\bar{\mu}-\underline{\mu} \leq \kappa$, from Lemma 2, the gap between the obtained utility and the optimal utility is bounded by $P_{\text {Con }} \kappa$. Moreover, $\lim _{\underline{\mu} \rightarrow \bar{\mu}} \hat{U}_{\text {CSRA }}(\underline{\mu}, \bar{\mu})=U_{\text {CSRA }}^{*}$. 
TABLE I: Algorithmic implementations of the proposed algorithms

\begin{tabular}{|c|c|}
\hline Proposed CSRA algorithm & Brute force algorithm for a given $\boldsymbol{I}$ \\
\hline 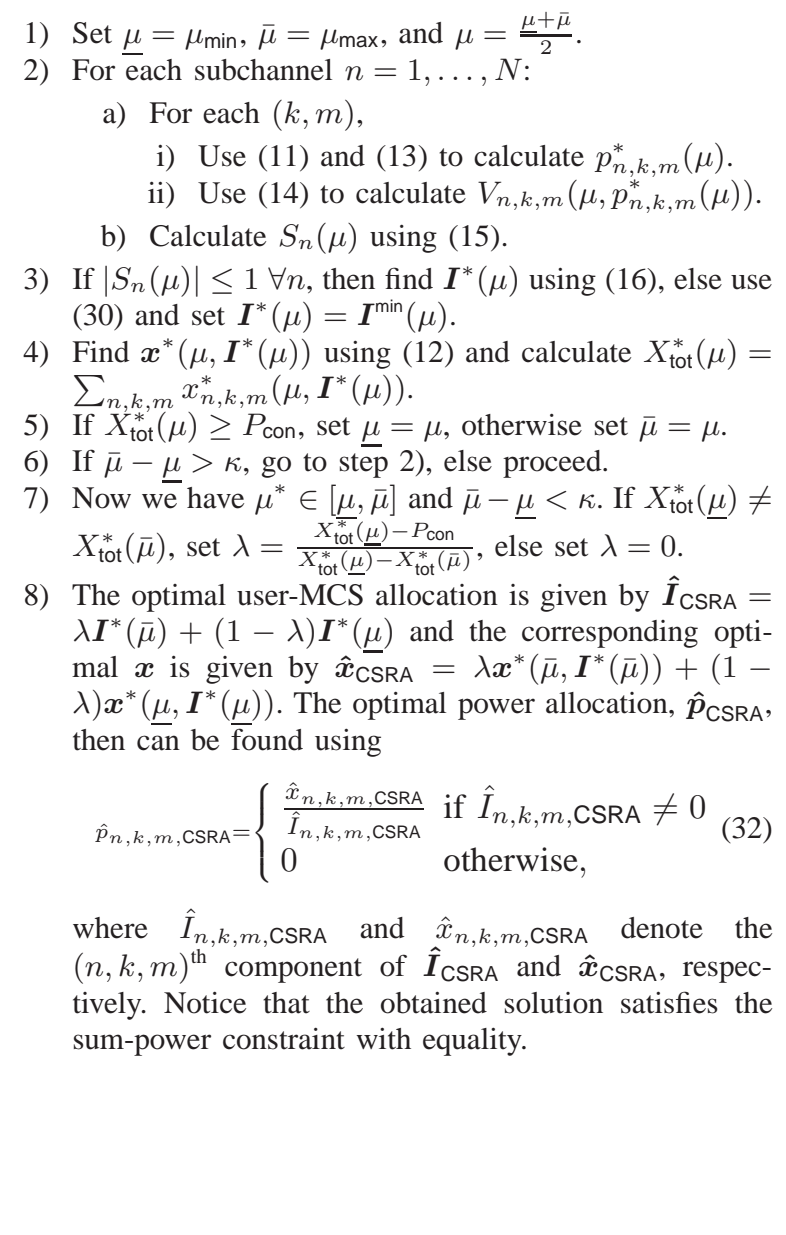 & 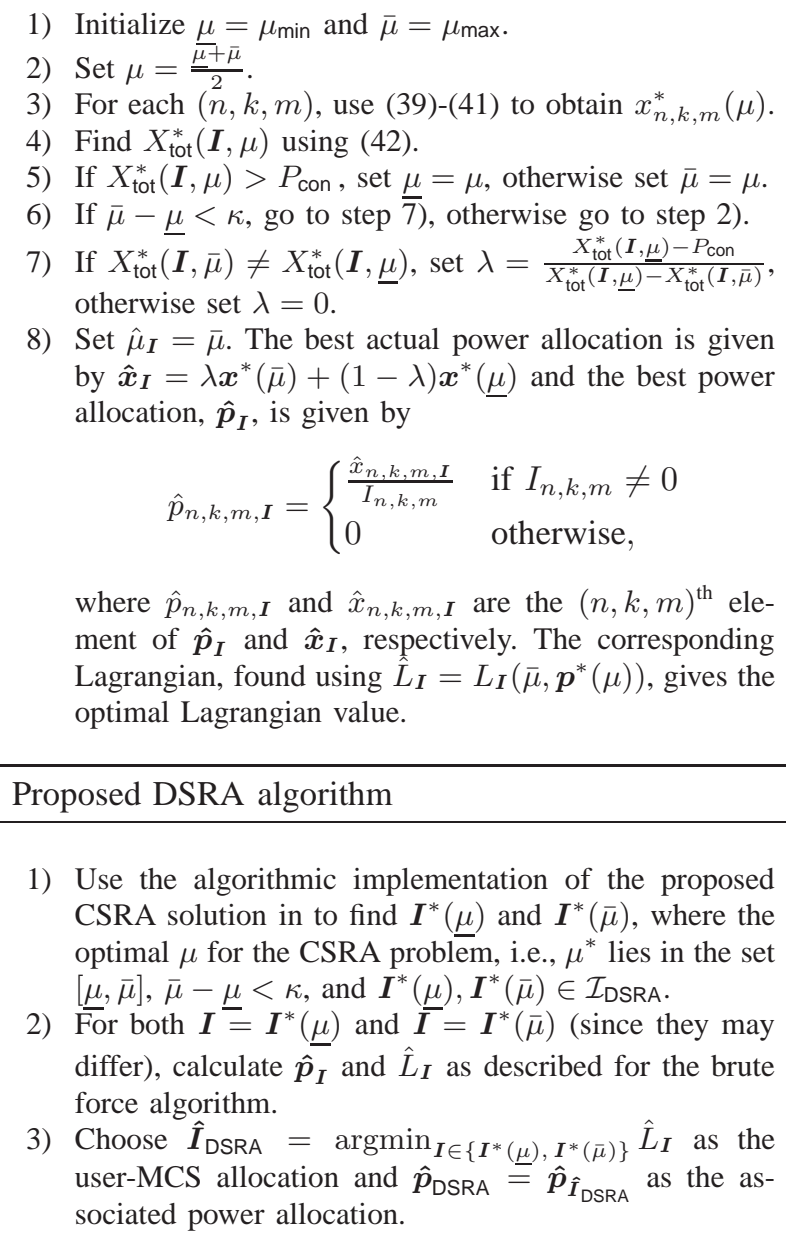 \\
\hline
\end{tabular}

The proposed algorithm requires at most $\left\lceil\log _{2}\left(\frac{\mu_{\max }-\mu_{\min }}{\kappa}\right)\right\rceil$ iterations of $\mu$ in order to find $\bar{\mu}$, and $\underline{\mu}$ such that $\bar{\mu}-\underline{\mu} \leq \kappa$ and $\mu^{*} \in[\underline{\mu}, \bar{\mu}]$. Therefore, measuring the complexity of the algorithm by the number of times (11) must be solved for a given $(n, k, m, \mu)$, the proposed algorithm takes at most

$$
N K M\left\lceil\log _{2}\left(\frac{\mu_{\max }-\mu_{\min }}{\kappa}\right)\right\rceil
$$

steps. We use this method of measuring complexity because it allows us to easily compare all algorithms in the paper. Note that, for a given $\kappa$, the number of steps taken by the proposed bisection algorithm is proportional to $\log _{2} \kappa$. 


\section{E. Some properties of the CSRA solution}

In this subsection, we study a few properties of the CSRA solution that yield valuable insights into the optimal resource allocation strategy for any given value of Lagrange multiplier, $\mu$. Let us fix a $\tilde{\mu} \in\left[\mu_{\min }, \mu_{\max }\right]$. Now, if $\left|S_{n}(\tilde{\mu})\right| \leq 1, \forall n$, then the optimal allocation at $\tilde{\mu}, \boldsymbol{I}^{*}(\tilde{\mu})$, is given by (16), which reveals that $\boldsymbol{I}^{*}(\tilde{\mu}) \in\{0,1\}^{N \times K \times M}$. In this case, the definition of $\mathcal{I}_{\text {CSRA }}$ implies that every subchannel is allocated to at most one user-MCS combination. Note that this is precisely the constraint we impose in the later part of this paper. Let us now consider the case where it is possible that $\left|S_{n}(\tilde{\mu})\right|>1$ for some $n$.

Lemma 3. For any $\tilde{\mu}>0$, there exists a $\delta>0$ such that for all $\mu \in(\tilde{\mu}-\delta, \tilde{\mu}+\delta) \backslash\{\tilde{\mu}\}$, there exists an

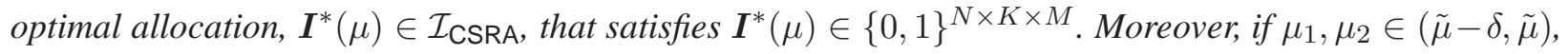
then there exists $\boldsymbol{I}^{*}\left(\mu_{1}\right), \boldsymbol{I}^{*}\left(\mu_{2}\right) \in\{0,1\}^{N \times K \times M}$ such that $\boldsymbol{I}^{*}\left(\mu_{1}\right)=\boldsymbol{I}^{*}\left(\mu_{2}\right)$. The same property holds if both $\mu_{1}, \mu_{2} \in(\tilde{\mu}, \tilde{\mu}+\delta)$.

Proof: A proof sketch is given in Appendix D For the full proof, see [19].

In conjunction with (12), the above lemma implies that the discontinuities in Fig. 3 are isolated and that, around every point on the horizontal axis, there is a small region over which $X_{\text {tot }}^{*}(\mu)$ is continuous. Hence, the number of such discontinuities are, at most, countable.

\section{Scheduling And Resource Allocation without subchannel Sharing}

In this section, we will solve the scheduling and resource allocation (SRA) problem (1) under the constraint that $I_{n, k, m} \in\{0,1\}$, i.e., that each subchannel can be allocated to at most one combination of user and MCS per time slot. We will refer to this problem as the "discrete scheduling and resource allocation" (DSRA) problem. Storing the values of $I_{n, k, m}$ in the $N \times K \times M$ matrix $\boldsymbol{I}$, the DSRA subchannel constraint can be expressed as $I \in \mathcal{I}_{\text {DSRA, where }}$

$$
\mathcal{I}_{\mathrm{DSRA}}:=\left\{\boldsymbol{I}: \boldsymbol{I} \in\{0,1\}^{N \times K \times M}, \sum_{k, m} I_{n, k, m} \leq 1 \forall n\right\} .
$$

Then, using (1), the DSRA problem can be stated as

DSRA $:=\max _{\substack{\left\{p_{n, k, m} \geq 0\right\} \\ \boldsymbol{I} \in \mathcal{I}_{\text {DSRA }}}} \sum_{n, k, m} I_{n, k, m} \mathrm{E}\left\{U_{n, k, m}\left(\left(1-a_{k, m} e^{-b_{k, m} p_{n, k, m} \gamma_{n, k}}\right) r_{k, m}\right)\right\}$ s.t. $\sum_{n, k, m} I_{n, k, m} p_{n, k, m} \leq P_{\text {con }}$.

Let us denote the optimal $\boldsymbol{I}$ and $\boldsymbol{p}$ for (34) by $\boldsymbol{I}_{\mathrm{DSRA}}^{*}$ and $\boldsymbol{p}_{\mathrm{DSRA}}^{*}$, respectively.

The DSRA problem is a mixed-integer programming problem. Mixed-integer programming problems are generally NP-hard, meaning that polynomial-time solutions do not exist [20]. Fortunately, in some 
cases, such as ours, one can exploit the problem structure to design polynomial-complexity algorithms that reach solutions in close vicinity of the exact solution. We first describe an approach to solve the DSRA mixed-integer programming problem exactly by exhaustively searching over all possible user-MCS allocations in order to arrive at the optimal user, rate, and power allocation. We will see that this "bruteforce" approach has a complexity that grows exponentially in the number of subchannels. Later, we will exploit the DSRA problem structure, and its relation to the CSRA problem, to design an algorithm with near-optimal performance and polynomial complexity.

\section{A. Brute-force algorithm}

Consider that, if we attempted to solve our DSRA problem via brute-force (i.e., by solving the power allocation sub-problem for every possible choice of $I \in \mathcal{I}_{\text {DSRA }}$ ), we would solve the following subproblem for every given $\boldsymbol{I}$.

$$
\max _{\left\{p_{n, k, m} \geq 0\right\}} \sum_{n, k, m} I_{n, k, m} \mathrm{E}\left\{U_{n, k, m}\left(\left(1-a_{k, m} e^{-b_{k, m} p_{n, k, m} \gamma_{n, k}}\right) r_{k, m}\right)\right\} \text { s.t. } \sum_{n, k, m} I_{n, k, m} p_{n, k, m} \leq P_{\text {con }} \text {. }
$$

Borrowing our approach to the CSRA problem, we could transform the variable $p_{n, k, m}$ into $x_{n, k, m}$ via the relation: $x_{n, k, m}=I_{n, k, m} p_{n, k, m}$. The problem in (35) can, therefore, be written as:

$$
\min _{\left\{x_{n, k, m} \geq 0\right\}} \sum_{n, k, m} I_{n, k, m} F_{n, k, m}\left(I_{n, k, m}, x_{n, k, m}\right) \text { s.t. } \sum_{n, k, m} x_{n, k, m} \leq P_{\mathrm{con}}
$$

where $F_{n, k, m}\left(I_{n, k, m}, x_{n, k, m}\right)$ is defined in (4). This problem is a convex optimization problem that satisfies Slater's condition [18] when $x_{n, k, m}=P_{\mathrm{con}} / 2 N K M$ for all $n, k, m$. Therefore, its solution is equal to the solution of its dual problem (i.e., zero duality gap) [18]. To formulate the dual problem, we write the Lagrangian of the primal problem (36) as

$$
L_{\boldsymbol{I}}(\mu, \boldsymbol{x})=\sum_{n, k, m} I_{n, k, m} F_{n, k, m}\left(I_{n, k, m}, x_{n, k, m}\right)+\left(\sum_{n, k, m} x_{n, k, m}-P_{\mathrm{con}}\right) \mu,
$$

where $\mu$ is the dual variable and $\boldsymbol{x}$ is the $N \times K \times M$ matrix containing actual powers allocated to all $(n, k, m)$ combinations. Note that the Lagrangian in (37) is exactly the same as the Lagrangian for the CSRA problem in (5). Using (37), the dual of the brute-force problem can be written as

$$
\max _{\mu \geq 0} \min _{\boldsymbol{x} \succeq 0} L_{\boldsymbol{I}}(\mu, \boldsymbol{x})=\max _{\mu \geq 0} L_{\boldsymbol{I}}\left(\mu, \boldsymbol{x}^{*}(\mu)\right)=L_{\boldsymbol{I}}\left(\mu_{\boldsymbol{I}}^{*}, \boldsymbol{x}^{*}\left(\mu_{\boldsymbol{I}}^{*}\right)\right),
$$

for optimal solutions $\mu_{\boldsymbol{I}}^{*}$ and $\boldsymbol{x}^{*}\left(\mu_{\boldsymbol{I}}^{*}\right)$. Minimizing $L_{\boldsymbol{I}}(\mu, \boldsymbol{x})$ over $\{\boldsymbol{x} \succeq 0\}$ by equating the differential of $L_{\boldsymbol{I}}(\mu, \boldsymbol{x})$ w.r.t. $x_{n, k, m}$ to zero (which is identical to the approach taken in Section $\amalg$ I-A for the CSRA problem), we get that, for any subchannel $n$,

$$
x_{n, k, m}^{*}(\mu)=I_{n, k, m} p_{n, k, m}^{*}(\mu) .
$$


Here,

$$
p_{n, k, m}^{*}(\mu)= \begin{cases}\tilde{p}_{n, k, m}(\mu) & \text { if } 0 \leq \mu \leq a_{k, m} b_{k, m} r_{k, m} U_{n, k, m}^{\prime}\left(\left(1-a_{k, m}\right) r_{k, m}\right) \mathrm{E}\left\{\gamma_{n, k}\right\} \\ 0 & \text { otherwise, }\end{cases}
$$

and $\tilde{p}_{n, k, m}(\mu)$ is the unique 3 value satisfying (11), repeated as (41) for convenience.

$$
\mu=a_{k, m} b_{k, m} r_{k, m} \mathrm{E}\left\{U_{n, k, m}^{\prime}\left(\left(1-a_{k, m} e^{-b_{k, m} \tilde{p}_{n, k, m}(\mu) \gamma_{n, k}}\right) r_{k, m}\right) \gamma_{n, k} e^{-b_{k, m} \tilde{p}_{n, k, m}(\mu) \gamma_{n, k}}\right\} .
$$

Note that the Lagrangian as well as the power allocation in (37) and 39) are identical to that obtained for the CSRA problem in (5) and (12), respectively. Also recall that (19)-(21) hold even when $I^{*}(\mu)$ is replaced by arbitrary $\boldsymbol{I}$. Thus, we have $\mu_{\boldsymbol{I}}^{*} \in\left[\mu_{\min }, \mu_{\max }\right]$, where $\mu_{\min }$ and $\mu_{\min }$ are defined in (20) and (21), respectively.

As discussed in Section $\llbracket-\mathrm{A}, \tilde{p}_{n, k, m}(\mu)$ is a strictly-decreasing continuous function of $\mu$, which makes $p_{n, k, m}^{*}(\mu)$ a decreasing continuous function of $\mu$. Let us now define

$$
X_{\text {tot }}^{*}(\boldsymbol{I}, \mu) \triangleq \sum_{n, k, m} x_{n, k, m}^{*}(\mu)=\sum_{n, k, m} I_{n, k, m} p_{n, k, m}^{*}(\mu)
$$

as the total optimal power allocation for allocation $\boldsymbol{I}$ at $\mu$. Therefore, $X_{\text {tot }}^{*}(\boldsymbol{I}, \mu)$ is also a decreasing continuous function of $\mu$. This reduces our problem to finding the minimum value of $\mu \in\left[\mu_{\min }, \mu_{\max }\right]$ for which $X_{\text {tot }}^{*}(\boldsymbol{I}, \mu)=P_{\text {con }}$. Such a problem structure (i.e., finding the minimum Lagrange multiplier satisfying a sum-power constraint) yields a water-filling solution (e.g., [9], [21]). To obtain such a solution (in our case, $\mu_{I}^{*}$ ) one can use the bisection-search algorithm given in Table \.

While there are many ways to find $\mu$, we focus on bisection-search for easy comparison to the CSRA algorithm. Then, to solve the resource allocation problem for a given $\boldsymbol{I} \in \mathcal{I}_{\mathrm{DSRA}}$, the complexity, in terms of the number of times (41) (or (11)) is solved to yield $\hat{\mu}_{\boldsymbol{I}}$ such that $\left|\hat{\mu}_{\boldsymbol{I}}-\mu_{\boldsymbol{I}}^{*}\right|<\kappa$, is $\left(\sum_{n, k, m} I_{n, k, m}\right)\left\lceil\log _{2}\left(\frac{\mu_{\max }-\mu_{\min }}{\kappa}\right)\right\rceil$. Since the brute-force algorithm examines $\left|\mathcal{I}_{\text {DSRA }}\right|=(K M+1)^{N}$ hypotheses of $\boldsymbol{I}$, the corresponding complexity needed to find the exact DSRA solution is $\left\lceil\log _{2}\left(\frac{\mu_{\max }-\mu_{\min }}{\kappa}\right)\right\rceil \times$ $\sum_{n=1}^{N} n\left(\begin{array}{l}N \\ n\end{array}\right)(K M)^{n}$ or, equivalently,

$$
\left\lceil\log _{2}\left(\frac{\mu_{\max }-\mu_{\min }}{\kappa}\right)\right\rceil \times(K M+1)^{N-1} N K M .
$$

Because this "brute-force" algorithm may be impractical to implement for practical values of $K, M$, and $N$, we focus, in the sequel, on lower-complexity DSRA approximations. In doing so, we exploit insights previously gained from our study of the CSRA problem.

\footnotetext{
${ }^{3}$ By assumption, $U_{n, k, m}^{\prime}(\cdot)$ is a decreasing positive function and $e^{-b_{k, m} \tilde{p}_{n, k, m}(\mu) \gamma_{n, k}}$ is a strictly-decreasing positive function of $\tilde{p}_{n, k, m}(\mu)$, which makes the right side of 411 a strictly-decreasing positive function of $\tilde{p}_{n, k, m}(\mu)$.
} 


\section{B. Proposed DSRA algorithm}

Equation (30) in Section 1 II-B demonstrated that there exists an optimal user-MCS allocation for the CSRA problem that either lies in the domain of DSRA problem, i.e., $\boldsymbol{I}^{*}\left(\mu^{*}\right) \in \mathcal{I}_{\mathrm{DSRA}}$, or is a convex combination of two points from the domain of DSRA problem, i.e., $\boldsymbol{I}^{*}\left(\mu^{*}\right)=\lambda \boldsymbol{I}^{\min }\left(\mu^{*}\right)+(1-$ $\lambda) \boldsymbol{I}^{\max }\left(\mu^{*}\right)$, where $\boldsymbol{I}^{\min }\left(\mu^{*}\right) \neq \boldsymbol{I}^{\max }\left(\mu^{*}\right)$ and $\boldsymbol{I}^{\min }\left(\mu^{*}\right), \boldsymbol{I}^{\max }\left(\mu^{*}\right) \in \mathcal{I}_{\text {DSRA }}$. Note that if $\boldsymbol{I} \in \mathcal{I}_{\text {CSRA }}$ and $\boldsymbol{I} \in\{0,1\}^{N \times K \times M}$, then $\boldsymbol{I} \in \mathcal{I}_{\text {DSRA }}$.) This observation motivates us to attack the DSRA problem using the CSRA algorithm. In this section, we provide the details of such an approach.

The following lemma will be instrumental in understanding the relationship between the CSRA and DSRA problems and will serve as the basis for allocating resources in the DSRA problem setup.

Lemma 4. If the solution of the Lagrangian dual of the CSRA problem (6) for a given $\mu$ is such that $I^{*}(\mu) \in\{0,1\}^{N \times K \times M}$, and the corresponding total power is $X_{\mathrm{tot}}^{*}(\mu)$ as in (31), then the solution to the optimization problem

$$
\begin{aligned}
& \left(\mathbb{P}^{*}, \mathbb{I}^{*}\right)=\underset{\substack{\boldsymbol{I} \in \mathcal{I}_{\mathrm{DSRA}} \\
\operatorname{argmax}}}{n, k, m} \mathbb{I}_{n, k, m} \mathrm{E}\left\{U _ { n , k , m } \left(\left(1-a_{k, m} e^{\left.\left.\left.-b_{k, m} \mathbb{P}_{n, k, m} \gamma_{n, k}\right) r_{k, m}\right)\right\} \text { s.t. } \sum_{n, k, m} \mathbb{I}_{n, k, m} \mathbb{P}_{n, k, m} \leq X_{\mathrm{tot}}^{*}(\mu)}\right.\right.\right. \\
& \text { satisfies } \mathbb{I}^{*}=\boldsymbol{I}^{*}(\mu) \text { and, for every }(n, k, m), \mathbb{P}_{n, k, m}^{*}= \begin{cases}\frac{x_{n, k, m}^{*}\left(\mu, \boldsymbol{I}^{*}(\mu)\right)}{I_{n, k, m}^{*}(\mu)} & \text { if } I_{n, k, m}^{*}(\mu) \neq 0 \\
0 & \text { otherwise. }\end{cases}
\end{aligned}
$$

Proof: A proof sketch is given in Appendix E For the full proof, see [19].

From the above lemma, we conclude that if a $\mu$ exists such that $\boldsymbol{I}^{*}(\mu) \in \mathcal{I}_{\text {DSRA }}$ and $X_{\text {tot }}^{*}(\mu)=P_{\text {con }}$, then the DSRA problem is solved exactly by the CSRA solution $\left(\boldsymbol{I}^{*}(\mu), \boldsymbol{x}^{*}\left(\mu, \boldsymbol{I}^{*}(\mu)\right)\right)$, i.e., the optimal user-MCS allocation $\boldsymbol{I}_{\mathrm{DSRA}}^{*}$ equals $\boldsymbol{I}^{*}(\mu)$ and the optimal power allocation, $\boldsymbol{p}_{\mathrm{DSRA}}^{*}$, for any $(n, k, m)$, is

$$
p_{n, k, m, \text { DSRA }}^{*}= \begin{cases}\frac{\left.x_{n, k, m}^{*}\left(\mu, \boldsymbol{I}^{*}(\mu)\right)\right)}{I_{n, k, m}^{*}(\mu)} & \text { if } I_{n, k, m}^{*}(\mu) \neq 0 \\ 0 & \text { otherwise. }\end{cases}
$$

Recall that the optimal total power achieved for a given value of Lagrange multiplier $\mu$, i.e., $X_{\text {tot }}^{*}(\mu)=$ $\sum_{n, k, m} x_{n, k, m}^{*}\left(\mu, \boldsymbol{I}^{*}(\mu)\right)$, is piece-wise continuous and that a discontinuity (or "gap") occurs at $\mu$ when multiple allocations achieving the same optimal value of Lagrangian exist. When the sum-power constraint, $P_{\text {con }}$, lies in one of those "gaps," the optimal allocation for the CSRA problem equals a convex combination of two elements from the set $\mathcal{I}_{\text {DSRA }}$, and the CSRA solution is not admissible for DSRA. In such cases, we are motivated to choose the approximate DSRA solution $\hat{\boldsymbol{I}}_{\mathrm{DSRA}} \in\left\{\boldsymbol{I}^{\min }(\mu), \boldsymbol{I}^{\max }(\mu)\right\}$ yielding highest utility. In Table I, we detail an implementation of our proposed DSRA algorithm that has significantly lower complexity than brute-force. The numerical simulations in Section $\nabla$ show that its 
performance is very close to optimal. Moreover, the following lemma bounds the asymptotic difference in utility achieved by the exact DSRA solution and that produced by our proposed DSRA algorithm.

Lemma 5. Let $\mu^{*}$ be the optimal $\mu$ for the CSRA problem and $\underline{\mu}, \bar{\mu}$ be such that $\mu^{*} \in[\underline{\mu}, \bar{\mu}]$. Let $U_{\mathrm{DSRA}}^{*}$ and $\hat{U}_{\mathrm{DSRA}}(\underline{\mu}, \bar{\mu})$ be the utilities achieved by the exact DSRA solution and the proposed DSRA algorithm, respectively. Then,

$$
\begin{aligned}
0 \leq U_{\mathrm{DSRA}}^{*}-\lim _{\underline{\mu} \rightarrow \bar{\mu}} \hat{U}_{\mathrm{DSRA}}(\underline{\mu}, \bar{\mu}) & \leq\left(\mu^{*}-\mu_{\min }\right)\left(P_{\mathrm{con}}-X_{\mathrm{tot}}^{*}\left(\boldsymbol{I}^{\min }\left(\mu^{*}\right), \mu^{*}\right)\right) \\
& \leq\left\{\begin{array}{ll}
0 & \text { if }\left|S_{n}\left(\mu^{*}\right)\right| \leq 1 \forall n \\
\left(\mu_{\max }-\mu_{\min }\right) P_{\text {con }} & \text { otherwise }
\end{array} .\right.
\end{aligned}
$$

Proof: The proof is given in Appendix E

It is interesting to note that the bound (46) does not scale with number of users $K$ or subchannels $N$. The complexity of the proposed DSRA algorithm is marginally greater than that of the CSRA algorithm, since an additional comparison of two possible user-MCS allocation choices is involved. In units of solving (11) for a given $(n, k, m, \mu)$, the DSRA complexity is at most

$$
N(K M+2)\left\lceil\log _{2}\left(\frac{\mu_{\max }-\mu_{\min }}{\kappa}\right)\right\rceil .
$$

Comparing (43) and (47), we find that the complexity of the proposed DSRA algorithm is polynomial in $N, K, M$, which is considerably less than that of the brute-force algorithm (i.e., exponential in $N$ ).

\section{Discussion}

Before concluding this section, we make some remarks about our approach to DSRA and its connections to CSRA. First, we note that the DSRA problem is an integer-programming problem due to the discrete domain $\{0,1\}$ assumed for $I_{n, k, m}$. Because integer programming problems are generally NP-hard (recall our "brute force" DSRA solution), one is strongly motivated to find a polynomial-complexity method whose performance is as high as possible. One possible approach is based on "relaxation," whereby the discrete domain is relaxed to an interval domain, the relaxed problem is solved (with polynomial complexity), and the obtained solution is mapped back to the discrete domain. In fact, relaxation was previously employed for OFDMA frequency-scheduling in [9], [11], and the DSRA approximation that we propose in Section IV-B can also be interpreted as a form of relaxation.

The optimization literature suggests that relaxation is successful in some—but not all—cases, implying that relaxation-based OFDMA algorithms must be designed with care. For example, relaxation has widely 
used to solve linear integer programs (LIPs) [22]-[24]. The DSRA, however, is a mixed-integer nonlinear program (MINLP), and for such problems relaxation does not always perform well [24], [25]. Now, one could cite the analysis in [26, p. 371], which shows that-for a broad class of integer programming problems - the duality gap goes to zero as the number of integer variables goes to infinity, to suggest that the DSRA problem can be well approximated by its relaxed counterpart, CSRA, as the number of OFDMA subchannels $N \rightarrow \infty$. However, in practice, the number of subchannels $N$ is often quite small, preventing the application of this argument. For example, in LTE systems [6], [27], each subchannel consists of 12 subcarriers, so that only 25 subchannels are used for $5 \mathrm{MHz}$ bandwidths, and only 6 are used for 1.4 MHz bandwidths.

The above considerations have motivated us to investigate, in detail, the relationship between the continuous and discrete resource allocation scenarios. The results of our investigation include insights into the dissimilarity between CSRA and DSRA solutions (e.g., Lemma 3 and Lemma 4), and an efficient polynomial-complexity DSRA approximation that (as we shall see in Section V) performs near-optimally for all $N$ and admits the tight performance bound (46).

\section{Numerical Evaluation}

In this section, we analyze the performance of an OFDMA downlink system that uses the proposed CSRA and DSRA algorithms for scheduling and resource allocation under different system parameters. Unless otherwise specified, we use the sum-goodput utility $U_{n, k, m}(g)=g$.

For downlink transmission, the BS employs an uncoded $2^{m+1}$-QAM signaling scheme with MCS index $m \in\{1, \ldots, 15\}$. In this case, we have $r_{k, m}=m+1$ bits per symbol and one symbol per codeword. In the error rate model $\epsilon_{k, m}(p \gamma)=a_{k, m} e^{-b_{k, m} p \gamma}$, we choose $a_{k, m}=1$ and $b_{k, m}=1.5 /\left(2^{m+1}-1\right)$ because the actual symbol error rate of a $2^{m+1}$-QAM system is proportional to $\exp \left(-1.5 p \gamma /\left(2^{m+1}-1\right)\right)$ in the high- $(p \gamma)$ regime [28] and is $\approx 1$ when $p \gamma=0$. We use the standard OFDM model [29] to describe the (instantaneous) frequency-domain observation made by the $k^{\text {th }}$ user on the $n^{\text {th }}$ subchannel:

$$
y_{n, k}=h_{n, k} x_{n}+\nu_{n, k}, \text { for } n \in\{1, \ldots, N\} \text { and } k \in\{1, \ldots, K\}
$$

In (48), $x_{n}$ denotes the QAM symbol broadcast by the BS on the $n^{\text {th }}$ subchannel, $h_{n, k}$ the gain of the $n^{\text {th }}$ subchannel between the $k^{\text {th }}$ user and the BS, and $\nu_{n, k}$ a corresponding complex Gaussian noise sample. We assume that $\left\{\nu_{n, k}\right\}$ is unit variance and white across $(n, k)$, and we recall that the exogenous subchannel-SNR satisfies $\gamma_{n, k}=\left|h_{n, k}\right|^{2}$. We furthermore assume that the $k^{\text {th }}$ user's

frequency-domain channel gains $\boldsymbol{h}_{k}=\left(h_{1, k}, \ldots, h_{N, k}\right)^{T} \in \mathbb{C}^{N}$ are related to its channel impulse response 
$\boldsymbol{g}_{k}=\left(g_{1, k}, \ldots, g_{L, k}\right)^{T} \in \mathbb{C}^{L}$ via $\boldsymbol{h}_{k}=\boldsymbol{F} \boldsymbol{g}_{k}$, where $\boldsymbol{F} \in \mathbb{C}^{N \times L}$ contains the first $L(<N)$ columns of the $N$-DFT matrix, and where $\left\{g_{l, k}\right\}$ are i.i.d. over $(l, k)$ and drawn from a zero-mean complex Gaussian distribution with variance $\sigma_{g}^{2}$ chosen so that $\mathrm{E}\left\{\gamma_{n, k}\right\}=1$. Since the total available power for all subchannels at the base-station is $P_{\text {con }}$, the average available SNR per subchannel will be denoted by $\mathrm{SNR}=\frac{P_{\text {con }}}{N} \mathrm{E}\left\{\gamma_{n, k}\right\}$

To model imperfect CSI, we assume that there is a channel-estimation period during which the mobiles take turns to each broadcast one pilot OFDM symbol, from which the BS estimates the corresponding subchannel gains. Furthermore, we assume that the channels do not vary between pilot and data periods. To estimate $\boldsymbol{h}_{k}$, we assume that the BS observes $\tilde{\boldsymbol{y}}_{k}=\sqrt{p_{\text {pilot }}} \boldsymbol{h}_{k}+\tilde{\boldsymbol{\nu}}_{k} \in \mathbb{C}^{N}$. Note that the average SNR per subchannel under pilot transmission is $\mathrm{SNR}_{\text {pilot }}=p_{\text {pilot }} \mathrm{E}\left\{\gamma_{n, k}\right\}$. The channel $\boldsymbol{h}_{k}$ and the pilot observations $\tilde{\boldsymbol{y}}_{k}$ are zero-mean jointly Gaussian, and furthermore $\boldsymbol{h}_{k} \mid \tilde{\boldsymbol{y}}_{k}$ is Gaussian with mean

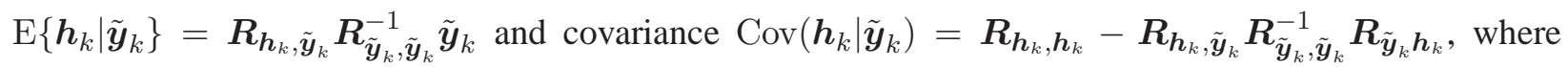
$\boldsymbol{R}_{\boldsymbol{z}_{1}, \boldsymbol{z}_{2}}$ denotes the cross-correlation of random vectors $\boldsymbol{z}_{1}$ and $\boldsymbol{z}_{2}$ [30, pp. 155]. Since $\boldsymbol{R}_{\boldsymbol{h}_{k}, \boldsymbol{h}_{k}}=\sigma_{g}^{2} \boldsymbol{F} \boldsymbol{F}^{\prime}$, $\boldsymbol{R}_{\boldsymbol{h}_{k}, \tilde{\boldsymbol{y}}_{k}}=\sqrt{p_{\text {pilot }}} \sigma_{g}^{2} \boldsymbol{F} \boldsymbol{F}^{\prime}$, and $\boldsymbol{R}_{\tilde{\boldsymbol{y}}_{k}, \tilde{\boldsymbol{y}}_{k}}=p_{\text {pilot }} \sigma_{g}^{2} \boldsymbol{F} \boldsymbol{F}^{\prime}+\mathbf{I}$ (where I denotes the identity matrix), it is straightforward to show that the elements on the diagonal of $\operatorname{Cov}\left(\boldsymbol{h}_{k} \mid \tilde{\boldsymbol{y}}_{k}\right)$ are equal. Furthermore, $\mathrm{E}\left\{\boldsymbol{h}_{k} \mid \tilde{\boldsymbol{y}}_{k}\right\}$ can be recognized as the pilot-aided MMSE estimate of $\boldsymbol{h}_{k}$. In summary, conditioned on the pilot observations, $h_{n, k}$ is Gaussian with mean $\hat{h}_{n, k}$ given by the $n^{\text {th }}$ element of $\mathrm{E}\left\{\boldsymbol{h}_{k} \mid \tilde{\boldsymbol{y}}_{k}\right\}$, and with variance $\sigma_{e}^{2}$ given by the first diagonal element of $\operatorname{Cov}\left(\boldsymbol{h}_{k} \mid \tilde{\boldsymbol{y}}_{k}\right)$. Thus, conditioned on the pilot observations, $\gamma_{n, k}$ has a non-central chi-squared distribution with two degrees of freedom.

We will refer to the proposed CSRA and DSRA algorithms implemented under imperfect CSI as "CSRA-ICSI" and "DSRA-ICSI," respectively. Their performances will be compared to that of "CSRAPCSI," i.e., CSRA implemented under perfect CSI, which serves as a performance upper bound, and fixed-power random-user scheduling (FP-RUS), which serves as a performance lower bound. FP-RUS schedules, on each subchannel, one user selected uniformly from $\{1, \ldots, K\}$, to which it allocates power $P_{\text {con }} / N$ and the fixed MCS $m$ that maximizes expected goodput. Unless specified, the number of OFDM subchannels is $N=64$, the number of users is $K=16$, the impulse response length is $L=2$, the average SNR per subchannel is $\mathrm{SNR}=10 \mathrm{~dB}$, the pilot $\mathrm{SNR}$ is $\mathrm{SNR}_{\text {pilot }}=-10 \mathrm{~dB}$, and the DSRA/CSRA tuning parameter is $\kappa=0.3 / P_{\text {con }}($ recall Table $\llbracket$ ). In all plots, goodput values were empirically averaged over 1000 realizations.

Figure 4 plots the subchannel-averaged goodput achieved by the above-described scheduling and resource-allocation schemes for different grades of CSI. In this curve, SNR estimates of subchannel SNR with different grades of accuracy. All other parameters remain unchanged. 

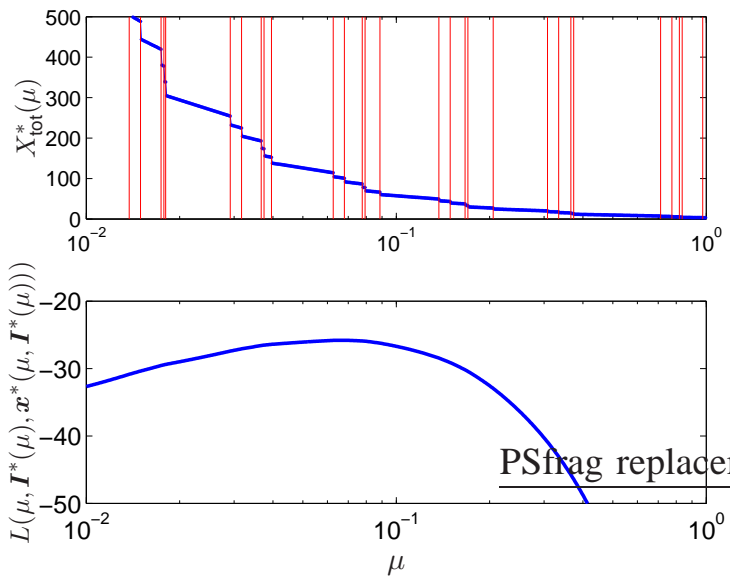

Fig. 3: Prototypical plot of $X_{\text {tot }}^{*}(\mu)$ and $L\left(\mu, \boldsymbol{I}^{*}(\mu), \boldsymbol{x}^{*}\left(\mu, \boldsymbol{I}^{*}(\mu)\right)\right)$ as a function of $\mu$ for $N=K=5$, and $P_{\text {con }}=100$. (See Section $\nabla$ for details.) The red vertical lines in the top plot show that a change in $I^{*}(\mu)$ occurs at that $\mu$.

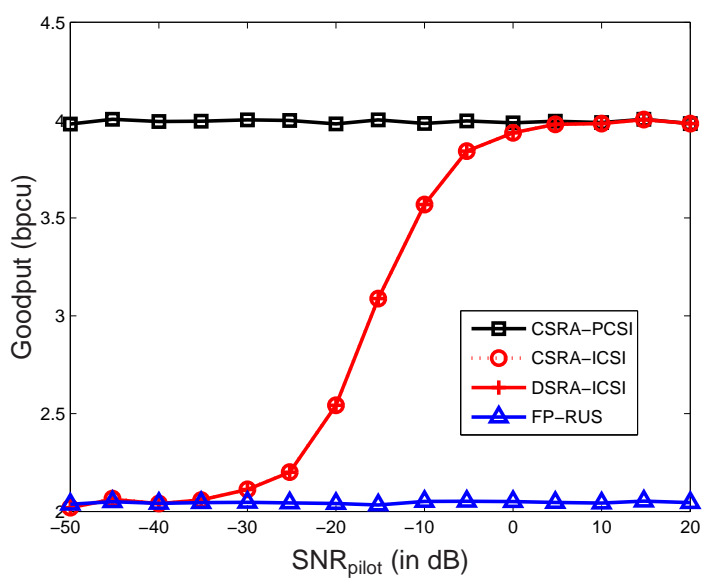

Fig. 4: Average goodput per subchannel versus $S N R_{\text {pilot }}$. Here, $N=64, K=16$, and $\mathrm{SNR}=10 \mathrm{~dB}$.

The plot shows that, as SNR pilot is increased, the performance of the proposed schemes (under the availability of imperfect CSI) increases from that of FP-RUS to that achieved by CSRA-PCSI. This is expected because, with increasing $\mathrm{SNR}_{\text {pilot}}$, the BS uses more accurate channel-state information for scheduling and resource allocation, and thus achieves higher goodput. The plot also shows that, even though the proposed CSRA algorithm exactly solves the CSRA problem and the proposed DSRA algorithm approximately solves the DSRA problem, their performances almost coincide. In particular, although the goodput achieved by CSRA-ICSI scheme exceeded that of DSRA-ICSI scheme in up-to $49 \%$ of the realizations, the maximum difference in the subchannel-averaged goodput was merely $4 \times 10^{-3}$ bits per channel-use (bpcu). Since the DSRA-ICSI schemes cannot achieve a sum-goodput higher than that achieved by the CSRA-ICSI scheme, it can be deduced that the proposed DSRA algorithm is exhibiting near-optimal performance.

Figure 5 plots the subchannel-averaged goodput versus the number of available users, $K$, ranging between 1 and 32. It shows that, as $K$ increases, the goodput per subchannel achieved by the proposed schemes increase under both perfect and imperfect CSI, whereas that achieved by the FP-RUS scheme remains constant. This is because, in the former case, the availability of more users can be exploited to schedule users with stronger subchannels, whereas, in the FP-RUS scheme, users are scheduled without regard to the instantaneous channel conditions. Similar to the observations in the previous plots, the performance difference between the proposed CSRA and DSRA algorithms remains negligible. In particular, although the goodput achieved by CSRA-ICSI exceeded that of DSRA-ICSI in up-to $29 \%$ of 


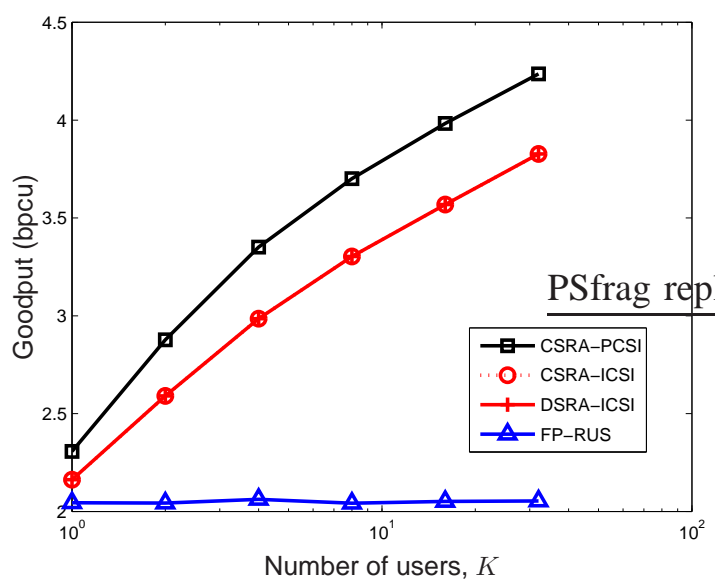

Fig. 5: Average goodput per subchannel versus number of users, $K$. In this plot, $N=64$, SNR $=10 \mathrm{~dB}$, and SNR $_{\text {pilot }}=-10 \mathrm{~dB}$.
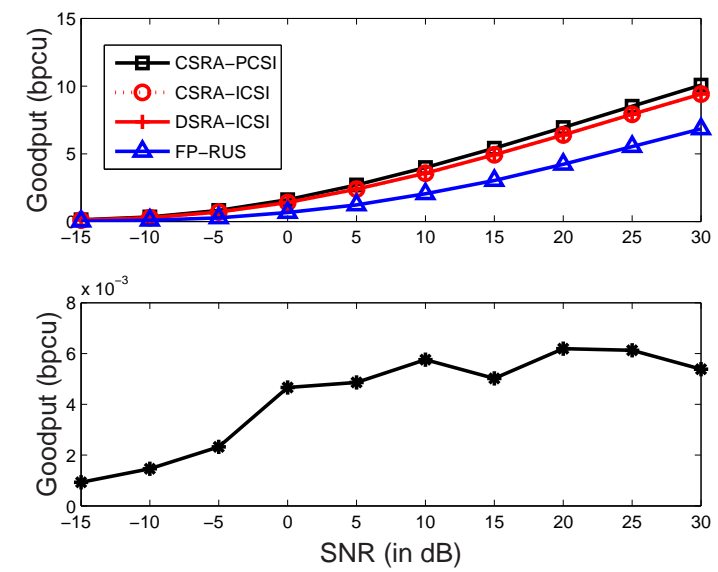

Fig. 6: The top plot shows the average goodput per subchannel as a function of SNR. The bottom plot shows the average bound on the optimality gap between the proposed and exact DSRA solutions (given in 45), i.e., the average value of $\left(\mu^{*}-\mu_{\min }\right)\left(P_{\text {con }}-X_{\text {tot }}^{*}\left(\boldsymbol{I}^{\min }, \mu^{*}\right)\right) / N$. In this plot, $N=64, K=16$, and $\mathrm{SNR}_{\text {pilot }}=-10 \mathrm{~dB}$.

the realizations, the maximum difference in the subchannel-averaged goodput was merely $7 \times 10^{-4} \mathrm{bpcu}$.

In Figure 6, the top plot shows the subchannel-averaged goodput and the bottom plot shows the subchannel and realization-averaged value of the bound (45) on the DSRA-ICSI optimality gap as a function of SNR. In the top plot, it can be seen that, as SNR increases, the difference between CSRA-PCSI and CSRA-ICSI (or, DSRA-ICSI) increases. However, the difference grows slower than the difference between CSRA-PCSI and FP-RUS. Interestingly, even for high values of SNR, the performance of CSRA-ICSI and DSRA-ICSI remain almost identical. In particular, although the goodput achieved by CSRA-ICSI scheme exceeded that of DSRA-ICSI scheme in up-to $28 \%$ of the realizations, the maximum difference in the subchannel-averaged goodput was merely $4 \times 10^{-5}$ bpcu. The bottom plot, which illustrates the average value of $\left(\mu^{*}-\mu_{\min }\right)\left(P_{\text {con }}-X_{\text {tot }}^{*}\left(\boldsymbol{I}^{\min }, \mu^{*}\right)\right)$ over all realizations and subchannels w.r.t. SNR, shows that the loss in sum-goodput over all subchannels due to the sub-optimality of proposed DSRA solution under imperfect CSI is bounded by $7 \times 10^{-3}$ bpcu, even when the subchannel-averaged goodput of DSRA-ICSI is of the order of tens of bpcu. These results confirm that the bound 45) is quite tight at high SNR.

Figure 7 shows the performance of the proposed DSRA algorithm under a sum-utility criterion that is motivated by a common pricing model for an elastic application such as file-transfer [3], [4]. In particular, we partitioned the $K=16$ users into two classes: $k \in\{1, \ldots, 8\} \triangleq \mathcal{K}_{1}$ is "Class 1 " and $k \in\{9, \ldots, 16\} \triangleq \mathcal{K}_{2}$ is "Class 2 ," and we ran DSRA with the utility $U_{k}(g) \triangleq\left(1-e^{-w_{1} g}\right) \mathbf{1}_{k \in \mathcal{K}_{1}}+$ 

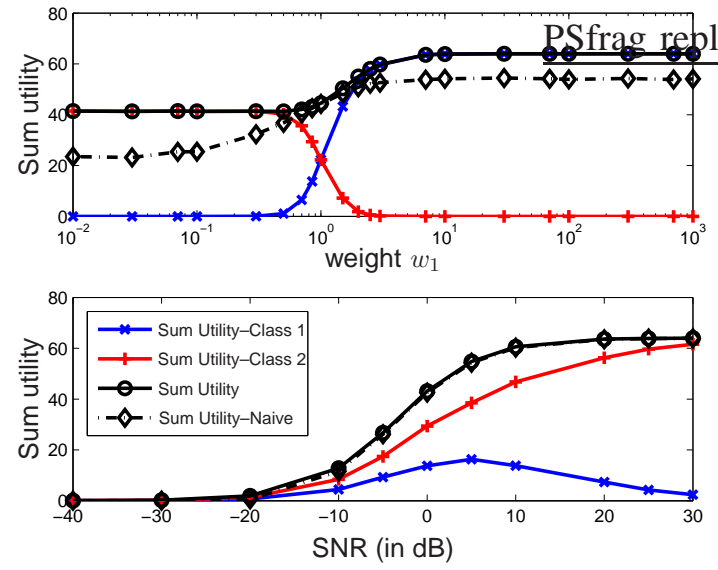

Fig. 7: The top plot shows sum utility versus $w_{1}$ when $w_{2}=1, \mathrm{SNR}=0 \mathrm{~dB}$. The bottom plot shows the sumutility versus SNR when $w_{1}=0.85, w_{2}=1$. Here, $N=$ $64, K=16$, and $\mathrm{SNR}_{\text {pilot }}=-10 \mathrm{~dB}$.
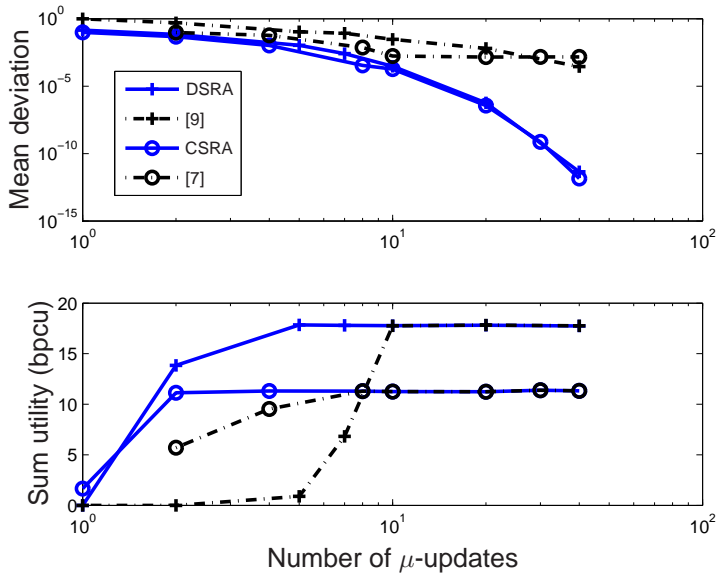

Fig. 8: The top plot shows the mean deviation of the estimated dual variable $\mu$ from $\mu^{*}$, and the bottom plot shows average sum-utility, as a function of the number of $\mu$-updates. Here, $N=64, K=16$, SNR $=10 \mathrm{~dB}$, and $\mathrm{SNR}_{\text {pilot }}=-10 \mathrm{~dB}$.

$\left(1-e^{-w_{2} g}\right) \mathbf{1}_{k \in \mathcal{K}_{2}}$, where $\mathbf{1}_{\mathcal{E}}$ denotes the indicator of event $\mathcal{E}$. The utility can be regarded as the revenue earned by the operator: when $w_{i}>w_{j}$, Class- $i$ users pay more (for a given goodput $g$ ) than Class- $j$ users in exchange for priority service. In Fig. 7 we show the resulting DSRA-maximized utility summed over all users, as well as that summed over each individual user class. For comparison, we show the utility (summed over all users) when DSRA is "naively" used to maximize sum-goodput instead of sum-utility. The top plot in Fig. 7 shows performance as a function of $w_{1}$, for fixed $w_{2}=1$ and $\mathrm{SNR}=0 \mathrm{~dB}$. There the behavior is as expected: when $w_{1} \ll w_{2}=1$ (i.e., Class- 1 users pay much less) DSRA allocates the overwhelming majority of the resources to Class-2 users, in an effort to earn more revenue. Meanwhile, when $w_{1} \gg w_{2}=1$, the overwhelming majority of resources are allocated to Class-1 users. Moreover, it is evident that the naive goodput-maximizing scheme does not earn the operator as much revenue as the utility-maximizing scheme (outside of the trivial case that $w_{1}=w_{2}$ ). The bottom plot in Fig. 7 shows the above described sum-utilities as a function of SNR, for fixed $w_{1}=0.85$ and $w_{2}=1$. There it can be seen that, at low SNR, the two classes achieve proportional utilities while, at high SNR, the utility of Class-1 users tend to zero. This behavior can be explained as follows: At low SNR, the goodputs $g$ are small, in which case $1-e^{-w_{i} g} \approx w_{i} g$, so that $U_{k}(g) \approx w_{i} g \mathbf{1}_{k \in \mathcal{K}_{i}}$, i.e., weighted-goodput utility. At high SNR, this approximation does not hold because the goodputs $g$ are usually large, and this particular pricing-based utility becomes increasingly unfair.

In Figure 8, we compare the performances of our proposed algorithms to the state-of-the-art algorithms in [7], [9]. In particular, we first compare the golden-section-search based algorithm from [7] to our CSRA 
algorithm. For CSRA, we choose the utility function and the SNR distributions to maximize the upper bound on capacity computed via the effective SNR $\frac{1}{K} \sum_{n, k} \log \left(1+\frac{p_{n, k, 1}\left|\hat{h}_{n, k}\right|^{4}}{\left|\hat{h}_{n, k}\right|^{2}+\sigma_{e}^{2} p_{n, k, 1}\left|\hat{h}_{n, k}\right|^{2}}\right)$ from [7, Eq. (4)]. Second, we compare the subgradient-based algorithm proposed for discrete allocation in [9] to our DSRA algorithm. For DSRA, we choose the utility $U_{n, k, m}(g)=\frac{1}{K} \log (1-\log (1-g)) \forall n, k, m$, so that we maximize $\frac{1}{K} \sum_{n, k} \mathrm{E}\left\{\log \left(1+p_{n, k, 1} \gamma_{n, k}\right)\right\}$, as in [9]. The top plot in Fig. 8 shows the mean deviation of the estimated value of the dual variable $\mu$ from the optimum (i.e., $\mu^{*}$ ), and the bottom plot shows the total utility achieved as a function of the number of $\mu$-updates. For the subgradient-based algorithm in [9], we set the step-size in the $i^{\text {th }} \mu$-update to be $1 / i$. In the top plot, it can be seen that the proposed algorithms outperform the algorithms in [7], [9] and converge toward $\mu^{*}$ at a much faster rate. The bottom plot shows that the proposed algorithms achieve a much higher utility than the algorithms in [7], [9] for the first few $\mu$-updates, illustrating the speed of our approaches. Note that the golden-section algorithm only provides estimates of $\mu^{*}$ at even numbers of $\mu$-updates.

\section{CONCLUSION}

In this paper, we considered the problem of joint scheduling and resource allocation (SRA) in downlink OFDMA systems under imperfect channel-state information. We considered two scenarios: 1) when subchannel sharing is allowed, and 2) when it is not. Both cases were framed as optimization problems that maximize a utility function subject to a sum-power constraint. Although the optimization problem in the first scenario (the so-called "continuous" or CSRA case) was found to be non-convex, we showed that it can be converted to a convex optimization problem and solved using a dual optimization approach with zero duality gap. An algorithmic implementation of the CSRA solution was also provided. The optimization problem faced in the second scenario (the so-called "discrete" or DSRA case) was found to be a mixed-integer programming problem. To attack it, we linked the DSRA problem to the CSRA problem, and showed that, in some cases, the DSRA solution coincides with the CSRA solution. For the case that the solutions do not coincide, we proposed a practical DSRA algorithm and bounded its performance. Numerical results were then presented under a variety of settings. The performance of the proposed CSRA and DSRA algorithms schemes under imperfect CSI were compared to those under perfect CSI and no instantaneous CSI (i.e., fixed-power random scheduling). In all cases, it was found that the proposed imperfect-CSI-based algorithms offer a significant advantage over schemes that do not use instantaneous CSI. Next, our DSRA bound was numerically evaluated and found to be extremely tight. We then demonstrated an application of DSRA to maximization of a pricing-based utility. Finally, our CSRA and DSRA algorithms were compared to the state-of-the-art golden-section-search [7] and 
subgradient [9] based algorithms and shown to yield significant improvements in convergence rate.

\section{REFERENCES}

[1] G. Song and Y. Li, "Utility-based resource allocation and scheduling in OFDM-based wireless broadband networks," IEEE Commun. Mag., vol. 43, pp. 127-134, Dec. 2005.

[2] M. Falkner, M. Devetsikiotis, and I. Lambadaris, "An overview of pricing concepts for broadband IP networks," IEEE Communications Surveys \& Tutorials, vol. 3, no. 2, 2000.

[3] L. A. DaSilva, "Pricing for QoS-enabled networks: A survey," IEEE Communications Surveys \& Tutorials, vol. 3, no. 2, 2000 .

[4] S. Shenker, "Fundamental design issues for the future internet," IEEE J. Select. Areas In Commun., vol. 13, pp. 1176 -1188 , Sep. 1995.

[5] “IEEE 802.16-2005 and IEEE Std 802.16-2004/Corl-2005," http://www.ieee802.org/16.

[6] “Overview of 3GPP Release 8 V0.2.2 (2011-01),” http://www.3gpp.org/ftp/Information/WORK_PLAN/Description_Releases/.

[7] J. Huang, V. Subramanian, R. Agrawal, and R. Berry, "Downlink scheduling and resource allocation for OFDM systems," IEEE Trans. Wireless Commun., vol. 8, pp. 288-296, Jan. 2009.

[8] “3GPP Technical Specifications (Release 10) 3GPP TS 25.101 V10.1.0,” http://www.3gpp.org/article/umts.

[9] I. C. Wong and B. L. Evans, "Optimal resource allocation in the OFDMA downlink with imperfect channel knowledge," IEEE Trans. Commun., vol. 57, pp. 232-241, Jan. 2009.

[10] G. Song and Y. Li, "Cross-layer optimization for OFDM wireless networks-Parts I and II," IEEE Trans. Wireless Commun., vol. 3, pp. 614-634, Mar. 2005.

[11] C. Y. Wong, R. S. Cheng, K. B. Letaief, and R. D. Murch, "Multiuser OFDM with adaptive subcarrier, bit and power allocation,” IEEE J. Select. Areas In Commun., vol. 17, pp. 1747-1758, Oct. 1999.

[12] T. J. Willink and P. H. Wittke, "Optimization and performance evaluation of multicarrier transmission," IEEE Trans. Inform. Theory, vol. 43, pp. 426-440, Mar. 1997.

[13] L. M. C. Hoo, B. Halder, J. Tellado, and J. M. Cioffi, "Multiuser transmit optimization for multicarrier broadcast channels: Asymptotic FDMA capacity region and algorithms," IEEE Trans. Commun., vol. 52, pp. 922-930, Jun. 2004.

[14] I. Wong and B. Evans, "Optimal downlink OFDMA resource allocation with linear complexity to maximize ergodic rates," IEEE Trans. Wireless Commun., vol. 7, pp. 962-971, Mar. 2008.

[15] K. Seong, M. Mohseni, and J. M. Cioffi, "Optimal resource allocation for OFDMA downlink systems," in Proc. IEEE Int. Symposium Inform. Theory, vol. 3, pp. 1394-1398, Jul. 2006.

[16] A. Ahmad and M. Assaad, "Margin adaptive resource allocation in downlink OFDMA system with outdated channel state information," Proc. IEEE Int. Symposium Personal Indoor Mobile Radio Commun., pp. 1868-1872, 2009.

[17] D. Hui and V. Lau, "Design and analysis of delay-sensitive cross-layer OFDMA systems with outdated CSIT," IEEE Trans. Wireless Commun., vol. 8, pp. 3484-3491, Jul. 2009.

[18] S. Boyd and L. Vandenberghe, Convex Optimization. Cambridge University Press, 2004.

[19] R. Aggarwal, On Scheduling and Resource Allocation using Imperfect Channel State Information. PhD thesis, The Ohio State University, Columbus, $\mathrm{OH}$, under preparation.

[20] J. Mitchell and E. K. Lee, "Branch-and-bound methods for integer programming," in Encyclopedia of Optimization (A. Floudas and P. M. Pardalos, eds.), vol. 2, ch. 4, pp. 509-519, The Netherlands: Kluwer Academic Publishers, 2001. 
[21] J. Jang and K. B. Lee, "Transmit power adaptation for multiuser OFDM systems," IEEE J. Select. Areas In Commun., vol. 21, pp. 171-178, Feb. 2003.

[22] M. Bénichou, J. M. Gauthier, P. Girodet, G. Hentges, G. Ribière, and O. Vincent, "Experiments in mixed-integer linear programming," Mathematical Programming, vol. 1, pp. 76-94, 1971.

[23] A. Lodi, "Mixed integer programming computation," in 50 Years of Integer Programming: 1958-2008 (M. Jünger et al., ed.), pp. 619-645, Berlin-Heidelberg: Springer-Verlag, 2010.

[24] G. Cornuejols, M. Conforti, and G. Zambelli, "Polyhedral approaches to mixed integer programming," in 50 Years of Integer Programming: 1958-2008 (M. Jünger et al., ed.), pp. 343-385, Berlin-Heidelberg: Springer-Verlag, 2010.

[25] R. C. Jeroslow, "There cannot be any algorithm for integer programming with quadratic constraints," Operations Research, vol. 21, no. 1, pp. 221-224, 1973.

[26] D. P. Bertsekas, Constrained Optimization and Lagrange Multiplier Methods. Academic Press, 1982.

[27] “UTRA-UTRAN Long Term Evolution (LTE) and 3GPP System Architecture Evolution (SAE) 3GPP LTA Paper," $3 G P P$. ftp://ftp.3gpp.org/Inbox/2008_web_files/LTA_Paper.pdf.

[28] J. G. Proakis, Digital Communications. New York: McGraw-Hill, 5th ed., 2008.

[29] J.-J. van de Beek, O. Edfors, M. Sandell, S. Wilson, and P. Borjesson, "On channel estimation in OFDM systems," in Proc. IEEE Veh. Tech. Conf., vol. 2, pp. 815-819, 1995.

[30] H. V. Poor, An Introduction to Signal Detection and Estimation. New York: Springer-Verlag, 2nd ed., 1994.

[31] H. Everett, "Generalized Lagrange multiplier method for solving problems of optimum allocation of resources," Operations Research, vol. 11, pp. 399-417, May-June 1963.

\section{APPENDIX A}

\section{SKETCH OF PROOF FOR CONVEXITY OF CSRA PROBLEM}

First, we show that $I_{n, k, m} F_{n, k, m}\left(I_{n, k, m}, x_{n, k, m}\right)$ is convex in $I_{n, k, m}$ and $x_{n, k, m}$. For this, consider the case when $I_{n, k, m}>0$. In this case, the Hessian of $I_{n, k, m} F_{n, k, m}\left(I_{n, k, m}, x_{n, k, m}\right)$ w.r.t. $I_{n, k, m}$ and $x_{n, k, m}$ can be calculated and found to be positive semi-definite. Next, consider the case when $I_{n, k, m}=0$. To prove convexity in this case, we apply the definition of convexity, i.e., for any two points $\left(I_{n, k, m}^{(1)}, x_{n, k, m}^{(1)}\right)$ and $\left(I_{n, k, m}^{(2)}, x_{n, k, m}^{(2)}\right)$ in the domain of CSRA problem and for any $\lambda \in[0,1]$, convexity means

$$
\begin{aligned}
& \lambda I_{n, k, m}^{(1)} F_{n, k, m}\left(I_{n, k, m}^{(1)}, x_{n, k, m}^{(1)}\right)+(1-\lambda) I_{n, k, m}^{(2)} F_{n, k, m}\left(I_{n, k, m}^{(2)}, x_{n, k, m}^{(2)}\right) \\
& \geq\left[\lambda I_{n, k, m}^{(1)}+(1-\lambda) I_{n, k, m}^{(2)}\right] F_{n, k, m}\left(\lambda I_{n, k, m}^{(1)}+(1-\lambda) I_{n, k, m}^{(2)}, \lambda x_{n, k, m}^{(1)}+(1-\lambda) x_{n, k, m}^{(2)}\right) .
\end{aligned}
$$

When one or both of $\left\{I_{n, k, m}^{(1)}, I_{n, k, m}^{(2)}\right\}$ are zero, it is straightforward to show that the above equation holds. Therefore, $I_{n, k, m} F_{n, k, m}\left(I_{n, k, m}, x_{n, k, m}\right)$ is convex in $I_{n, k, m}$ and $x_{n, k, m}$. Consequently, it is a convex function of $\boldsymbol{I}$ and $\boldsymbol{x}$. Since the primal objective function of the CSRA problem $\sum_{n, k, m} I_{n, k, m} F_{n, k, m}\left(I_{n, k, m}, x_{n, k, m}\right)$ is a sum of functions that are convex in $\boldsymbol{I}$ and $\boldsymbol{x}$, it is also convex in $\boldsymbol{I}$ and $\boldsymbol{x}$. 


\section{APPENDIX B}

\section{SKETCH OF PROOF OF LEMMA 1}

Suppose that $\mu_{1}<\mu_{2}$, where $\mu_{1}, \mu_{2} \in\left[\mu_{\min }, \mu_{\max }\right]$. With $\mu$ fixed, the minimization problem becomes

$$
\begin{aligned}
& L\left(\mu, \boldsymbol{I}^{*}(\mu), \boldsymbol{x}^{*}\left(\mu, \boldsymbol{I}^{*}(\mu)\right)\right) \\
& =\min _{\substack{\{\boldsymbol{x} \succeq 0\} \\
\boldsymbol{I} \in \mathcal{I}_{\text {CSRA }}}} L(\mu, \boldsymbol{I}, \boldsymbol{x})=\min _{\substack{\{\boldsymbol{x} \succeq 0\} \\
\boldsymbol{I} \in \mathcal{I}_{\text {CSRA }}}}\left(\sum_{n, k, m} x_{n, k, m}-P_{\text {Con }}\right) \mu+\sum_{n, k, m} I_{n, k, m} F_{n, k, m}\left(I_{n, k, m}, x_{n, k, m}\right)
\end{aligned}
$$

recalling (6). At $\mu=\mu_{1}, \boldsymbol{I}^{*}\left(\mu_{2}\right)$ and $\boldsymbol{x}^{*}\left(\mu_{2}, \boldsymbol{I}^{*}\left(\mu_{2}\right)\right)$ are suboptimal values of $\boldsymbol{I}^{*}(\mu)$ and $\boldsymbol{x}^{*}\left(\mu, \boldsymbol{I}^{*}(\mu)\right)$, and at $\mu=\mu_{2}, \boldsymbol{I}^{*}\left(\mu_{1}\right)$ and $\boldsymbol{x}^{*}\left(\mu_{1}, \boldsymbol{I}^{*}\left(\mu_{1}\right)\right)$ are suboptimal values of $\boldsymbol{I}^{*}(\mu)$ and $\boldsymbol{x}^{*}\left(\mu, \boldsymbol{I}^{*}(\mu)\right)$. Therefore,

$$
\begin{aligned}
& L\left(\mu_{1}, \boldsymbol{I}^{*}\left(\mu_{1}\right), \boldsymbol{x}^{*}\left(\mu_{1}, \boldsymbol{I}^{*}\left(\mu_{1}\right)\right)\right) \leq L\left(\mu_{1}, \boldsymbol{I}^{*}\left(\mu_{2}\right), \boldsymbol{x}^{*}\left(\mu_{2}, \boldsymbol{I}^{*}\left(\mu_{2}\right)\right)\right), \text { and } \\
& L\left(\mu_{2}, \boldsymbol{I}^{*}\left(\mu_{2}\right), \boldsymbol{x}^{*}\left(\mu_{2}, \boldsymbol{I}^{*}\left(\mu_{2}\right)\right)\right) \leq L\left(\mu_{2}, \boldsymbol{I}^{*}\left(\mu_{1}\right), \boldsymbol{x}^{*}\left(\mu_{1}, \boldsymbol{I}^{*}\left(\mu_{1}\right)\right)\right) .
\end{aligned}
$$

Adding (51) and 52), and evaluating the result, we get

$$
\left(\mu_{1}-\mu_{2}\right)\left(\sum_{n, k, m} x_{n, k, m}^{*}\left(\mu_{1}, \boldsymbol{I}^{*}\left(\mu_{1}\right)\right)-x_{n, k, m}^{*}\left(\mu_{2}, \boldsymbol{I}^{*}\left(\mu_{2}\right)\right)\right) \leq 0 .
$$

Since $\mu_{1}<\mu_{2}$, we have $X_{\text {tot }}^{*}\left(\mu_{1}\right) \geq X_{\text {tot }}^{*}\left(\mu_{2}\right)$. Therefore, $X_{\text {tot }}^{*}(\mu)$ is monotonically decreasing in $\mu$.

\section{APPENDIX C}

\section{Proof of LEMMa 2}

Proof: To compare the utilities obtained by the proposed CSRA algorithm and the exact CSRA solution, we compare the Lagrangian values achieved by the two solutions. Recall $\mu^{*} \in[\underline{\mu}, \bar{\mu}] \subset\left[\mu_{\min }, \mu_{\max }\right]$. Therefore,

$$
\begin{aligned}
& L\left(\mu^{*}, \boldsymbol{I}^{*}\left(\mu^{*}\right), \boldsymbol{x}^{*}\left(\mu^{*}, \boldsymbol{I}^{*}\left(\mu^{*}\right)\right)\right)-L\left(\underline{\mu}, \boldsymbol{I}^{*}(\underline{\mu}), \boldsymbol{x}^{*}\left(\underline{\mu}, \boldsymbol{I}^{*}(\underline{\mu})\right)\right) \geq 0, \text { and } \\
& L\left(\mu^{*}, \boldsymbol{I}^{*}\left(\mu^{*}\right), \boldsymbol{x}^{*}\left(\mu^{*}, \boldsymbol{I}^{*}\left(\mu^{*}\right)\right)\right)-L\left(\bar{\mu}, \boldsymbol{I}^{*}(\bar{\mu}), \boldsymbol{x}^{*}\left(\bar{\mu}, \boldsymbol{I}^{*}(\bar{\mu})\right)\right) \geq 0 .
\end{aligned}
$$

The solution of the proposed CSRA algorithm allocates resources such that the sum-power constraint is satisfied while achieving a Lagrangian value of

$$
\hat{L}_{\mathrm{CSRA}} \triangleq \lambda L\left(\bar{\mu}, \boldsymbol{I}^{*}(\bar{\mu}), \boldsymbol{x}^{*}\left(\bar{\mu}, \boldsymbol{I}^{*}(\bar{\mu})\right)\right)+(1-\lambda) L\left(\underline{\mu}, \boldsymbol{I}^{*}(\underline{\mu}), \boldsymbol{x}^{*}\left(\underline{\mu}, \boldsymbol{I}^{*}(\underline{\mu})\right)\right) \text {. }
$$

For any $\mu$, notice that $L\left(\mu, \boldsymbol{I}^{*}(\mu), \boldsymbol{x}^{*}\left(\mu, \boldsymbol{I}^{*}(\mu)\right)\right)=-U^{*}(\mu)+\left(X_{\text {tot }}^{*}(\mu)-P_{\text {con }}\right) \mu$, where $U^{*}(\mu)$ is the total utility achieved due to optimal power allocation at that $\mu$. Since the resource allocation obtained 
by the proposed CSRA algorithm and the exact CSRA solution satisfy the sum-power constraint with equality, we have

$$
\begin{aligned}
U_{\mathrm{CSRA}}^{*} & =-L\left(\mu^{*}, \boldsymbol{I}^{*}\left(\mu^{*}\right), \boldsymbol{x}^{*}\left(\mu^{*}, \boldsymbol{I}^{*}\left(\mu^{*}\right)\right)\right), \text { and } \\
\hat{L}_{\mathrm{CSRA}} & \left.=-\hat{U}_{\mathrm{CSRA}}(\underline{\mu}, \bar{\mu})+\left(X_{\mathrm{tot}}^{*}(\bar{\mu})-P_{\mathrm{Con}}\right) \lambda \bar{\mu}+\left(X_{\mathrm{tot}}^{*} \underline{\mu}\right)-P_{\mathrm{Con}}\right)(1-\lambda) \underline{\mu} \\
& =-\hat{U}_{\mathrm{CSRA}}(\underline{\mu}, \bar{\mu})+\left(X_{\mathrm{tot}}^{*}(\bar{\mu})-P_{\mathrm{Con}}\right)(\bar{\mu}-\underline{\mu}) \lambda .
\end{aligned}
$$

Equation (56) holds since $\lambda X_{\text {tot }}^{*}(\bar{\mu})+(1-\lambda) X_{\text {tot }}^{*}(\underline{\mu})=P_{\text {con. From (55) and (56), we get }}$

$$
0 \leq U_{\mathrm{CSRA}}^{*}-\hat{U}_{\mathrm{CSRA}}(\underline{\mu}, \bar{\mu})=-L\left(\mu^{*}, \boldsymbol{I}^{*}\left(\mu^{*}\right), \boldsymbol{x}^{*}\left(\mu^{*}, \boldsymbol{I}^{*}\left(\mu^{*}\right)\right)\right)+\hat{L}_{\mathrm{CSRA}}-\left(X_{\text {tot }}^{*}(\bar{\mu})-P_{\mathrm{Con}}\right)(\bar{\mu}-\underline{\mu}) \lambda .
$$

From the above equation and (54), we have

$$
0 \leq U_{\mathrm{CSRA}}^{*}-\hat{U}_{\mathrm{CSRA}}(\underline{\mu}, \bar{\mu}) \leq\left(P_{\mathrm{con}}-X_{\text {tot }}^{*}(\bar{\mu})\right)(\bar{\mu}-\underline{\mu}) \lambda \leq(\bar{\mu}-\underline{\mu}) P_{\mathrm{con}}
$$

\section{APPENDIX D}

\section{SKETCH OF PROOF OF LEMMA 3}

Let $\tilde{\mu} \in\left[\mu_{\min }, \mu_{\max }\right]$ be any value of the Lagrangian dual variable for the CSRA problem. Then, at $\tilde{\mu}$, one of the following three cases holds.

1) $\left|S_{n}(\tilde{\mu})\right| \leq 1 \forall n$.

2) For some $n,\left|S_{n}(\tilde{\mu})\right|>1$ but no two combinations in $S_{n}(\tilde{\mu})$ have the same allocated power.

3) For some $n,\left|S_{n}(\tilde{\mu})\right|>1$ and at least two combinations in $S_{n}(\tilde{\mu})$ have the same allocated power.

We make use of two properties in the proof. Firstly, $V_{n, k, m}\left(\mu, p_{n, k, m}^{*}(\mu)\right)$ is a continuous function of $\mu$. Therefore, by definition of continuous functions, if $V_{n, k, m}\left(\tilde{\mu}, p_{n, k, m}^{*}(\tilde{\mu})\right)>0$, then we can fix a $\delta_{n, k, m}(>0)$ such that $V_{n, k, m}\left(\mu, p_{n, k, m}^{*}(\mu)\right)>0$ whenever $|\mu-\tilde{\mu}|<\delta_{n, k, m}$. Secondly, for all values of $\mu$, we know $\frac{\partial V_{n, k, m}\left(\mu, p_{n, k, m}^{*}(\mu)\right)}{\partial \mu}=p_{n, k, m}^{*}(\mu)$. We now apply these properties to each of the three cases to determine $S_{n}(\mu) \forall n$. When $\mu$ is sufficiently close to $\tilde{\mu}$, we show that, in cases 1) and 2), one can fix a $\delta$ such that $\left|S_{n}(\mu)\right| \leq 1 \forall n$ whenever $0<|\mu-\tilde{\mu}|<\delta$. When this happens, it can be shown that, for all $\mu_{1}, \mu_{2} \in(\tilde{\mu}-\delta, \tilde{\mu})$, one has $\boldsymbol{I}^{*}\left(\mu_{1}\right), \boldsymbol{I}^{*}\left(\mu_{2}\right) \in\{0,1\}^{N \times K \times M}$ and $S_{n}\left(\mu_{1}\right)=S_{n}\left(\mu_{2}\right) \forall n$. The same property holds when $\mu_{1}, \mu_{2} \in(\tilde{\mu}, \tilde{\mu}+\delta)$. In case 3$)$, we establish that all combinations with the same allocated power contribute equally to the total power allocated, as well as the total optimal value of Lagrangian. Therefore, all but any one combination can be ignored safely, implying that there exists a fixed $\delta$ such that $\boldsymbol{I}^{*}(\mu) \in\{0,1\}^{N \times K \times M}$ whenever $|\mu-\tilde{\mu}|<\delta$. After ignoring the redundant 
combinations, it follows from cases 1) and 2) that, for all $\mu_{1}, \mu_{2} \in(\tilde{\mu}-\delta, \tilde{\mu})$ and $\mu_{1}, \mu_{2} \in(\tilde{\mu}, \tilde{\mu}+\delta)$, there exists $\boldsymbol{I}^{*}\left(\mu_{1}\right), \boldsymbol{I}^{*}\left(\mu_{2}\right) \in\{0,1\}^{N \times K \times M}$ such that $\boldsymbol{I}^{*}\left(\mu_{1}\right)=\boldsymbol{I}^{*}\left(\mu_{2}\right)$.

\section{APPENDIX E}

\section{SKETCH OF PROOF OF LEMMA 4}

From (6) and the stated assumptions, we have $\boldsymbol{I}^{*}(\mu) \in \mathcal{I}_{\text {DSRA }} \subset \mathcal{I}_{\text {CSRA }}$ and

$$
\left(\boldsymbol{I}^{*}(\mu), \boldsymbol{x}^{*}\left(\mu, \boldsymbol{I}^{*}(\mu)\right)\right)=\underset{\substack{\boldsymbol{x} \succeq 0 \\ \boldsymbol{I} \in \mathcal{I}_{\text {DSRA }}}}{\operatorname{argmin}} \sum_{n, k, m} I_{n, k, m} F_{n, k, m}\left(I_{n, k, m}, x_{n, k, m}\right)+\left(\sum_{n, k, m} x_{n, k, m}-P_{\text {con }}\right) \mu,
$$

where $F_{n, k, m}(\cdot, \cdot)$ was defined in (4). Then, applying the concept of generalized Lagrange multiplier method from [31, Theorem 1], we conclude that

$$
\left(\mathbb{I}^{*}, \mathbb{X}^{*}\right)=\underset{\substack{\{\mathbb{X} \succeq 0\} \\ \mathbb{I} \in \mathcal{I}_{\text {DSRA }}}}{\operatorname{argmin}} \sum_{n, k, m} \mathbb{I}_{n, k, m} F_{n, k, m}\left(\mathbb{I}_{n, k, m}, \mathbb{X}_{n, k, m}\right) \text { s.t. } \sum_{n, k, m} \mathbb{X}_{n, k, m} \leq \sum_{n, k, m} x_{n, k, m}^{*}\left(\mu, \boldsymbol{I}^{*}(\mu)\right) \text {. ( }
$$

Substituting $\mathbb{X}_{n, k, m}=\mathbb{I}_{n, k, m} \mathbb{P}_{n, k, m}$ back into the above equation, we obtain the desired result.

\section{APPENDIX F}

\section{PROOF OF LEMMA 5}

Proof: Let us denote $\lim _{\underline{\mu} \rightarrow \bar{\mu}} \hat{U}_{\text {DSRA }}(\underline{\mu}, \bar{\mu})$ by $\hat{U}_{\text {DSRA }}$. The left inequality in the lemma is straightforward since $U_{\mathrm{DSRA}}^{*} \geq \hat{U}_{\mathrm{DSRA}}(\underline{\mu}, \bar{\mu}) \forall \underline{\mu}, \bar{\mu}$. Now, if $\left|S_{n}\left(\mu^{*}\right)\right| \leq 1 \forall n$, then we have $U_{\mathrm{DSRA}}^{*}=U_{\mathrm{CSRA}}^{*}=$ $\hat{U}_{\text {DSRA }}$, ensuring that the solution obtained via the proposed DSRA algorithm is optimal in the limit $\underline{\mu}, \bar{\mu} \rightarrow \mu^{*}$. However, when $\left|S_{n}\left(\mu^{*}\right)\right|>1$ for some $n, P_{\text {con }}$ lies in one of the "gaps" as mentioned in

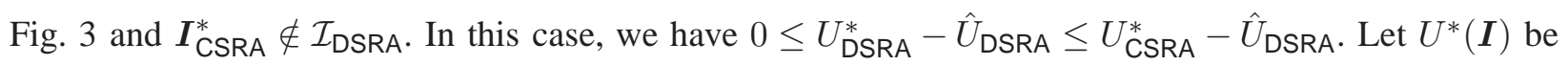

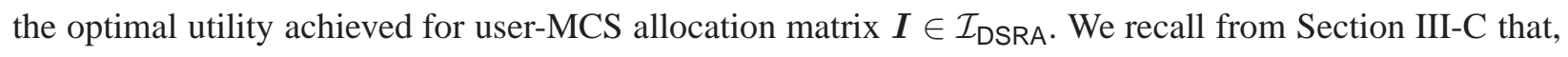
at $\mu^{*}$, the allocation $\boldsymbol{I}^{\min }\left(\mu^{*}\right)$ is one of possibly many values of $\boldsymbol{I}$ minimizing $L\left(\mu^{*}, \boldsymbol{I}, \boldsymbol{x}^{*}\left(\mu^{*}, \boldsymbol{I}\right)\right)$. Thus, $U_{\text {CSRA }}^{*}=-L\left(\mu^{*}, \boldsymbol{I}^{\min }\left(\mu^{*}\right), \boldsymbol{x}^{*}\left(\mu^{*}, \boldsymbol{I}^{\min }\left(\mu^{*}\right)\right)\right)$. For brevity in this proof, let us denote $\boldsymbol{I}^{\min }\left(\mu^{*}\right)$ and $\boldsymbol{I}^{\max }\left(\mu^{*}\right)$ $\left(\in \mathcal{I}_{\text {DSRA }}\right)$, defined in $\left(\underline{30}\right.$, by $\boldsymbol{I}^{\min }$ and $\boldsymbol{I}^{\max }$, respectively. Therefore, $\hat{U}_{\mathrm{DSRA}}=\max \left\{U^{*}\left(\boldsymbol{I}^{\min }\right), U^{*}\left(\boldsymbol{I}^{\max }\right)\right\}$. This gives us

$$
\begin{aligned}
U_{\mathrm{CSRA}}^{*}-\hat{U}_{\mathrm{DSRA}} & \leq U_{\mathrm{CSRA}}^{*}-U^{*}\left(\boldsymbol{I}^{\mathrm{min}}\right) \\
& \left.=-L\left(\mu^{*}, \boldsymbol{I}^{\min }, \boldsymbol{x}^{*}\left(\mu^{*}, \boldsymbol{I}^{\min }\right)\right)+L_{\boldsymbol{I}^{\min }}\left(\mu_{\boldsymbol{I}^{\min }}^{*}, \boldsymbol{x}^{*}\left(\mu_{\boldsymbol{I}^{\min }}^{*}\right)\right)\right) \\
& =-L\left(\mu^{*}, \boldsymbol{I}^{\min }, \boldsymbol{x}^{*}\left(\mu^{*}, \boldsymbol{I}^{\min }\right)\right)+L\left(\mu_{\boldsymbol{I}^{\min }}, \boldsymbol{I}^{\min }, \boldsymbol{x}^{*}\left(\mu_{\boldsymbol{I}^{\min }, \boldsymbol{I}^{\min }}\right)\right)
\end{aligned}
$$

where, for (60), we use the equivalence between $L(\mu, \boldsymbol{I}, \boldsymbol{x})$ in (5) and $L_{\boldsymbol{I}}(\mu, \boldsymbol{x})$ in (37). Note that $\mu_{\boldsymbol{I}^{\min }}^{*} \leq \mu^{*}$, since the total optimally allocated power for $\boldsymbol{I}^{\min }$ at $\mu=\mu^{*}$ is less than or equal to $P_{\text {con }}$ and 
the total optimally allocated power for any given $\boldsymbol{I}$ is a decreasing function of $\mu$. Plugging $L(\cdot, \cdot, \cdot)$ from (5) into (60), we get

$$
\begin{aligned}
U_{\text {CSRA }}^{*}-\hat{U}_{\text {DSRA }} \leq & -\left[-\mu^{*} P_{\text {Con }}+\sum_{n, k, m} I_{n, k, m}^{\min }\left(-\bar{U}_{n, k, m}\left(p_{n, k, m}^{*}\left(\mu^{*}\right)\right)+\mu^{*} p_{n, k, m}^{*}\left(\mu^{*}\right)\right)\right] \\
& +\left[-\mu_{\boldsymbol{I}^{\min }}^{*} P_{\text {con }}+\sum_{n, k, m} I_{n, k, m}^{\min }\left(-\bar{U}_{n, k, m}\left(p_{n, k, m}^{*}\left(\mu_{\boldsymbol{I}^{\min }}^{*}\right)\right)+\mu^{*}\left(\boldsymbol{I}^{\min }\right) p_{n, k, m}^{*}\left(\mu_{\boldsymbol{I}^{\min }}^{*}\right)\right)\right],
\end{aligned}
$$

where, $\bar{U}_{n, k, m}(x)=\mathrm{E}\left\{U_{n, k, m}\left(\left(1-a_{k, m} e^{-b_{k, m} x \gamma_{n, k}}\right) r_{k, m}\right)\right\}$. Using the definition of $X_{\text {tot }}^{*}(\boldsymbol{I}, \mu)$ in (42), we have $X_{\text {tot }}^{*}\left(\boldsymbol{I}^{\mathrm{min}}, \mu^{*}\right) \leq P_{\text {con }}$ and $X_{\text {tot }}^{*}\left(\boldsymbol{I}^{\min }, \mu_{\boldsymbol{I}^{\mathrm{min}}}^{*}\right)=P_{\text {con. }}$. Therefore, 61) can be re-written as

$$
\begin{aligned}
& U_{\text {CSRA }}^{*}-\hat{U}_{\text {DSRA }} \\
& \leq \mu^{*}\left(P_{\text {con }}-X_{\text {tot }}^{*}\left(\boldsymbol{I}^{\min }, \mu^{*}\right)\right)-\sum_{n, k, m} I_{n, k, m}^{\min }\left[\bar{U}_{n, k, m}\left(p_{n, k, m}^{*}\left(\mu_{\boldsymbol{I}^{\min }}^{*}\right)\right)-\bar{U}_{n, k, m}\left(p_{n, k, m}^{*}\left(\mu^{*}\right)\right)\right] .
\end{aligned}
$$

Calculating the first two derivatives of $\bar{U}_{n, k, m}(x)$ with respect to $x$, we find that it is a strictly-increasing concave function of $x$. Therefore, if $x_{1} \leq x_{2}$, one can write that $\bar{U}_{n, k, m}\left(x_{2}\right)-\bar{U}_{n, k, m}\left(x_{1}\right) \geq\left(x_{2}-\right.$ $\left.x_{1}\right) \bar{U}_{n, k, m}^{\prime}\left(x_{2}\right)$. Plugging $x_{1}=p_{n, k, m}^{*}\left(\mu^{*}\right)$ and $x_{2}=p_{n, k, m}^{*}\left(\mu_{I^{\text {min }}}^{*}\right)$ into this inequality, we get

$$
\bar{U}_{n, k, m}\left(p_{n, k, m}^{*}\left(\mu_{\boldsymbol{I}^{\min }}^{*}\right)\right)-\bar{U}_{n, k, m}\left(p_{n, k, m}^{*}\left(\mu^{*}\right)\right) \geq\left.\left(p_{n, k, m}^{*}\left(\mu_{\boldsymbol{I}^{\min }}^{*}\right)-p_{n, k, m}^{*}\left(\mu^{*}\right)\right) \frac{\partial \bar{U}_{n, k, m}(x)}{\partial x}\right|_{x=p_{n, k, m}^{*}\left(\mu_{I^{\min }}^{*}\right)} \text { (63) }
$$

From (62) and 63), we then get

$$
\begin{aligned}
& U_{\text {CSRA }}^{*}-\hat{U}_{\text {DSRA }} \\
& \leq \mu^{*}\left(P_{\text {con }}-X_{\text {tot }}^{*}\left(\boldsymbol{I}^{\min }, \mu^{*}\right)\right)-\sum_{n, k, m} I_{n, k, m}^{\min } \bar{U}_{n, k, m}^{\prime}\left(p_{n, k, m}^{*}\left(\mu_{\boldsymbol{I}^{\min }}^{*}\right)\right)\left(p_{n, k, m}^{*}\left(\mu_{\boldsymbol{I}^{\min }}^{*}\right)-p_{n, k, m}^{*}\left(\mu^{*}\right)\right) .
\end{aligned}
$$

Evaluating $\bar{U}_{n, k, m}^{\prime}\left(p_{n, k, m}^{*}\left(\mu_{\boldsymbol{I}^{\mathrm{min}}}^{*}\right)\right)$, we find

$$
\begin{aligned}
& \left.\frac{\partial \bar{U}_{n, k, m}(x)}{\partial x}\right|_{x=p_{n, k, m}^{*}\left(\mu_{I^{\min }}^{*}\right)}=a_{k, m} b_{k, m} r_{k, m} \mathrm{E}\left\{U_{n, k, m}^{\prime}\left(\left(1-a_{k, m} e^{-b_{k, m} p_{n, k, m}^{*}\left(\mu_{I \min }^{*}\right) \gamma_{n, k}}\right) r_{k, m}\right) \gamma_{n, k} e^{-b_{k, m} p_{n, k, m}^{*}\left(\mu_{I \min }^{*}\right) \gamma_{n, k}}\right\} \\
& \geq \mu_{\min } .
\end{aligned}
$$

From (64) and 65), we finally obtain

$$
U_{\text {CSRA }}^{*}-\hat{U}_{\text {DSRA }} \leq\left(\mu^{*}-\mu_{\min }\right)\left(P_{\text {con }}-X_{\text {tot }}^{*}\left(\boldsymbol{I}^{\min }, \mu^{*}\right)\right) \leq\left(\mu_{\max }-\mu_{\min }\right) P_{\text {con }} .
$$

